\title{
Almost flat K-theory of classifying spaces
}

\author{
José R. Carrión* \\ Department of Mathematics \\ Penn State University \\ University Park, PA, 16803 \\ United States \\ jcarrion@psu.edu
}

\author{
Marius Dadarlat ${ }^{\dagger}$ \\ Department of Mathematics \\ Purdue University \\ West Lafayette, IN, 47907 \\ United States \\ mdd@math.purdue.edu
}

August 3, 2021

\begin{abstract}
We give a rigorous account and prove continuity properties for the correspondence between almost flat bundles on a triangularizable compact connected space and the quasi-representations of its fundamental group. For a discrete countable group $\Gamma$ with finite classifying space $B \Gamma$, we study a correspondence between between almost flat K-theory classes on $B \Gamma$ and group homomorphism $K_{0}\left(C^{*}(\Gamma)\right) \rightarrow \mathbb{Z}$ that are implemented by pairs of discrete asymptotic homomorphisms from $C^{*}(\Gamma)$ to matrix algebras.
\end{abstract}

\section{Introduction}

Connes, Gromov and Moscovici [3] developed and used the concepts of almost flat bundle, almost flat K-theory class and group quasi-representation as tools for proving the Novikov conjecture for large classes of groups. For a compact manifold $M$, it was shown in [3] that the signature with coefficients in a (sufficiently) almost flat bundle is a homotopy invariant. Moreover, the authors indicate that they have a reformulation of the notion of almost flatness to bundles with infinite dimensional fibers which allows them to show that if $\Gamma$ is a countable discrete group such that all classes of $K^{0}(B \Gamma)$ are almost flat (up to torsion), then $\Gamma$ satisfies the Novikov conjecture $[3$, Sec. $6]$.

The problem of constructing nontrivial almost flat K-theory classes is interesting in itself. Suppose that the classifying space $B \Gamma$ of a countable discrete group $\Gamma$ admits a realization as a finite simplicial complex. Using results of Kasparov [13, $\mathrm{Yu}[24$ and $\mathrm{Tu}$ [23, the second-named author showed in [5] that if $\Gamma$ is coarsely embeddable in a Hilbert space and the full group $\mathrm{C}^{*}$-algebra $C^{*}(\Gamma)$ is quasidiagonal, then all classes in $K^{0}(B \Gamma)$ are almost flat.

Inspired by [3], in this paper we investigate the correspondence between between the almost flat classes in $K^{0}(B \Gamma)$ and the group homomorphisms $h: K_{0}\left(C^{*}(\Gamma)\right) \rightarrow \mathbb{Z}$ that are implemented by pairs of discrete asymptotic homomorphisms $\left\{\pi_{n}^{ \pm}: C^{*}(\Gamma) \rightarrow M_{k(n)}(\mathbb{C})\right\}_{n=1}^{\infty}$, in the sense that $h(x) \equiv$ $\left(\pi_{n}^{+}\right)_{\sharp}(x)-\left(\pi_{n}^{-}\right)_{\sharp}(x)$ for $x \in K_{0}\left(C^{*}(\Gamma)\right)$; see Definition 8.1. It turns out that this correspondence

\footnotetext{
* Partially supported by NSF Postdoctoral Fellowship \#DMS-1303884 and the Center for Symmetry and Deformation at the University of Copenhagen.

${ }^{\dagger}$ Partially supported by NSF grant \#DMS-1362824. 2010 Mathematics Subject Classification 46L05 (primary), 46L80, 46L85 (secondary)
} 
is one-to-one (modulo torsion) if the full assembly map $\mu: K^{0}(B \Gamma) \rightarrow K_{0}\left(C^{*}(\Gamma)\right)$ is bijective. See Theorem 8.7 and its generalization with coefficients Theorem 3.1 .

We take this opportunity to give a self-contained presentation of the correspondence (and its continuity properties) between almost flat bundles on a connected triangularizable compact space and the quasi-representations of its fundamental group; see Theorems 3.1, 3.3. As far as we can tell, while this correspondence was more or less known to the experts, it has not been well documented in the literature. We rely on work of Phillips and Stone [19,20]. These authors studied topological invariants associated with lattice gauge fields via a construction which associates an almost flat bundle to a lattice gauge field with controlled distortion and small modulus of continuity.

The following terminology is useful for further discussion of our results. If $\mathbf{k}=(k(n))_{n=1}^{\infty}$ is a sequence of natural numbers, we write $Q_{\mathbf{k}}=\prod_{n=1}^{\infty} M_{k(n)}(\mathbb{C}) / \sum_{n=1}^{\infty} M_{k(n)}(\mathbb{C})$. Recall that a separable $\mathrm{C}^{*}$-algebra $A$ is $\mathrm{MF}$ is it embeds as a $\mathrm{C}^{*}$-subalgebra of $Q_{\mathbf{k}}$ for some $\mathbf{k}$. In other words, there are sufficiently many $*$-homomorphisms $A \rightarrow Q_{\mathbf{k}}$ to capture the norm of the elements in $A$, 1]. By analogy, let us say that a separable $\mathrm{C}^{*}$-algebra $A$ is "K-theoretically MF" if there exist sufficiently many *-homomorphisms $A \rightarrow Q_{\mathbf{k}}$ to capture the K-theory of $A$ in the following sense: For any homomorphism $h: K_{0}\left(C^{*}(\Gamma)\right) \rightarrow \mathbb{Z}$ there exist $\mathbf{k}$ and two $*$-homomorphisms $\pi^{ \pm}: A \rightarrow Q_{\mathbf{k}}$ such that $\pi_{*}^{+}(x)-\pi_{*}^{-}(x)=h_{\infty}(x)$ for all $x \in K_{0}(A)$. Here we identify $K_{0}\left(Q_{\mathbf{k}}\right)$ with a subgroup of $\prod_{n=1}^{\infty} \mathbb{Z} / \sum_{n=1}^{\infty} \mathbb{Z}$, and $h_{\infty}(x)$ is the coset of element $(h(x), h(x), h(x), \ldots) \in \prod_{n=1}^{\infty} \mathbb{Z}$. With this terminology, Theorem 8.7 reads as follows:

Theorem. Let $\Gamma$ be a discrete countable group whose classifying space $B \Gamma$ is a finite simplicial complex. If the full assembly map $\mu: K_{0}(B \Gamma) \rightarrow K_{0}\left(C^{*}(\Gamma)\right)$ is bijective, then the following conditions are equivalent:

(1) All elements of $K^{0}(B \Gamma)$ are almost flat modulo torsion;

(2) $C^{*}(\Gamma)$ is K-theoretically $M F$.

By a result of Higson and Kasparov [11, the assumptions of this theorem are satisfied by groups $\Gamma$ with the Haagerup property and finite classifying spaces.

We note that a separable quasidiagonal $\mathrm{C}^{*}$-algebra that satisfies the UCT is K-theoretically MF by [5, Prop. 2.5]. We suspect that a similar result holds for MF algebras satisfying the UCT.

\section{Basic definitions and notation}

Let $X$ be a connected compact metric space that admits a finite triangulation. This means that $X$ is the geometric realization of some connected finite simplicial complex $\Lambda$, written $X=|\Lambda|$. Let $\Gamma=\pi_{1}(X)$ be the fundamental group of $X$. Let $A$ be a unital $C^{*}$-algebra. We establish a correspondence between quasi-representations of $\Gamma$ into $\mathrm{GL}(A)$ and almost flat bundles over $X$ with fiber $A$ and structure group GL(A). To be more accurate, this correspondence is between almost unitary quasi-representations and almost unitary principal bundles. It is convenient to work in a purely combinatorial context. Following [19], we call the combinatorial version of an almost flat bundle an almost flat coordinate bundle. See Definition 2.5. We prove the equivalence of these concepts in Proposition 7.2 .

For our combinatorial approach we begin with a finite simplicial complex $\Lambda$ endowed with some additional structure, as follows. The vertices of $\Lambda$ are denoted by $i, j, k$ with possible indices. The set of $k$-simplices of $\Lambda$ is denoted by $\Lambda^{(k)}$. We fix a maximal tree $T \subset \Lambda$ and a root vertex $i_{0}$. 


\section{The edge-path group and quasi-representations}

It will be convenient to view the fundamental group of the geometric realization of $\Lambda$ as the edgepath group $E\left(\Lambda, i_{0}\right)$ of $\Lambda$. The groups $\pi_{1}(|\Lambda|)$ and $E\left(\Lambda, i_{0}\right)$ are isomorphic and we will simply write $\Gamma$ for either group.

Once $\Lambda$ and $T$ are fixed so is the following standard presentation of $\Gamma$ in terms of the edges of $\Lambda$ (see e.g. [22, Sections 3.6-3.7]): $\Gamma$ is isomorphic to the group generated by the collection of all edges $\langle i, j\rangle$ of $\Lambda$ subject to the following relations:

- if $\langle i, j\rangle$ is an edge of $T$, then $\langle i, j\rangle=1$;

- if $i, j, k$ are vertices of a simplex of $\Lambda$, then $\langle i, j\rangle\langle j, k\rangle=\langle i, k\rangle$.

We should point out that in the second relation $i, j$, and $k$ are not necessarily distinct; consequently $\langle i, j\rangle\langle j, i\rangle=\langle i, i\rangle$. Since $\langle i, i\rangle=\langle i\rangle$ belongs to $T$ one has $\langle i, j\rangle^{-1}=\langle j, i\rangle$ as expected.

2.1 Notation. Let $\mathbb{F}_{\Lambda}$ be the free group generated by the edge set of $\Lambda$ and let $q: \mathbb{F}_{\Lambda} \rightarrow \Gamma$ be the group epimorphism corresponding the presentation of $\Gamma$ just described.

Write $\mathcal{F}_{\Lambda}$ for the image under $q$ of the edge set of $\Lambda$; this is a symmetric generating set for $\Gamma$. Write $\gamma_{i j}=q(\langle i, j\rangle)$ for the elements of $\mathcal{F}_{\Lambda}$. Let $\mathcal{R} \subset \mathbb{F}_{\Lambda}$ be the collection of all relators:

$$
\mathcal{R}:=\{\langle i, j\rangle \mid\langle i, j\rangle \text { is an edge of } T\} \cup\left\{\langle i, j\rangle\langle j, k\rangle\langle i, k\rangle^{-1} \mid i, j, k \text { are vertices of a simplex of } \Lambda\right\} .
$$

Choose a set-theoretic section $s: \Gamma \rightarrow \mathbb{F}_{\Lambda}$ of $q$ that takes the neutral element of $\Gamma$ to the neutral element of $\mathbb{F}_{\Lambda}$. This section $s$ will remain fixed for the rest of the paper.

We introduce one last notation before the definition. If $A$ is a unital $\mathrm{C}^{*}$-algebra and $\delta>0$, set

$$
\mathrm{U}(A)_{\delta}=\{v \in A: \operatorname{dist}(v, \mathrm{U}(A))<\delta\} .
$$

Note that $\mathrm{U}(A)_{\delta} \subseteq \mathrm{GL}(A)$ if $\delta<1$.

2.2 Definition. Let $A$ be a unital $\mathrm{C}^{*}$-algebra.

(1) Let $\mathcal{F} \subset \Gamma$ be finite and let $0<\delta<1$. A function $\pi: \Gamma \rightarrow \operatorname{GL}(A)$ is an $(\mathcal{F}, \delta)$-representation of $\Gamma$ if

(a) $\pi(\gamma) \in \mathrm{U}(A)_{\delta}$ for all $\gamma \in \mathcal{F}$;

(b) $\left\|\pi\left(\gamma \gamma^{\prime}\right)-\pi(\gamma) \pi\left(\gamma^{\prime}\right)\right\|<\delta$ for all $\gamma, \gamma^{\prime} \in \mathcal{F}$;

(c) $\pi(e)=1_{A}$, where $e$ is the neutral element of $\Gamma$.

(2) Define a pseudometric $d$ on the set of all bounded maps $\Gamma \rightarrow A$ by

$$
d\left(\pi, \pi^{\prime}\right)=\max _{\gamma \in \mathcal{F}_{\Lambda}}\left\|\pi(\gamma)-\pi^{\prime}(\gamma)\right\|
$$

We may sometimes refer to an $(\mathcal{F}, \delta)$-representation as a "quasi-representation" without specifying $\mathcal{F}$ or $\delta$. In most of the paper we will take $\mathcal{F}=\mathcal{F}_{\Lambda}$. 


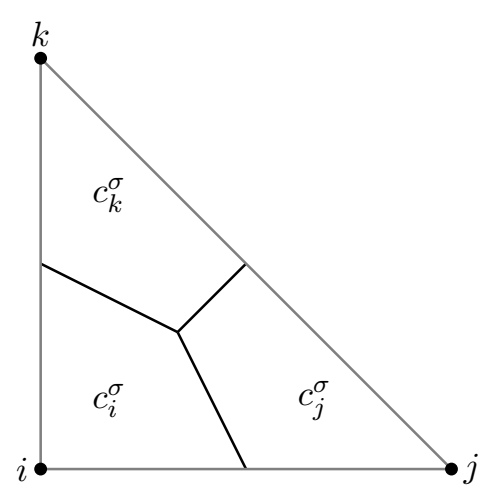

(a)

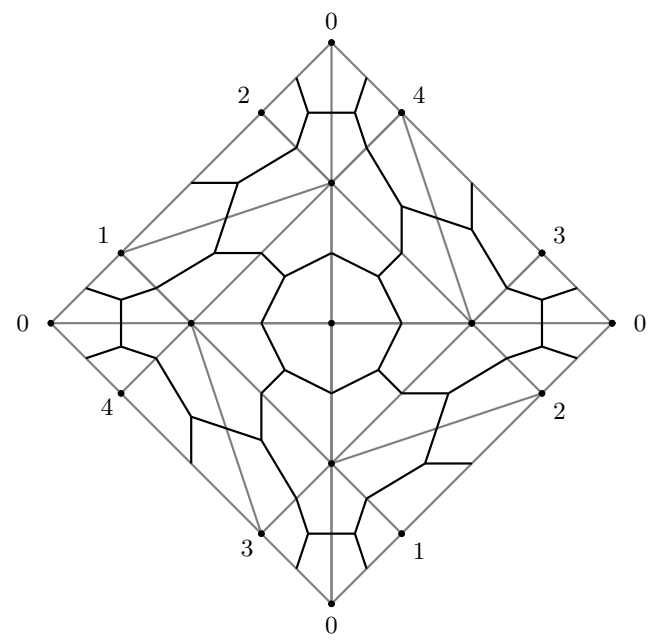

(b)

Figure 1: (a) Dual cell blocks in a simplex $\sigma=\langle i, j, k\rangle$. (b) A triangulation of $\mathbb{T}^{2}$ with the dual cell structure highlighted.

\section{The dual cover and almost flat coordinate bundles}

Once $\Lambda$ is fixed, so is a cover $\mathcal{C}_{\Lambda}$ of $|\Lambda|$, called the dual cover. We recall its definition (borrowing heavily from the appendix of $[20])$.

2.3 Definition. Let $\sigma=\langle 0, \ldots, r\rangle$ be a simplex of $\Lambda$. For $i \in\{0, \ldots, r\}$, the dual cell block $c_{i}^{\sigma}$, dual to $i$ in $\sigma$, is defined in terms of the barycentric coordinates $\left(t_{o}, \ldots, t_{r}\right)$ by

$$
c_{i}^{\sigma}=\left\{\left(t_{0}, \ldots, t_{r}\right) \mid t_{i} \geq t_{j} \text { for all } j\right\} \subset|\Lambda| .
$$

The dual cell $c_{i}$, dual to the vertex $i$, is the union of cell blocks dual to $i$ :

$$
c_{i}=\cup\left\{c_{i}^{\sigma} \mid i \in \sigma\right\} .
$$

The dual cover $\mathcal{C}_{\Lambda}$ is the collection of all dual cells. (See Figure 1.)

2.4 Notation. We usually write $c_{i j}$ for the intersection $c_{i} \cap c_{j}, c_{i j k}$ for $c_{i} \cap c_{j} \cap c_{k}$ etc. The barycenter of a simplex $\sigma$ is denoted $\hat{\sigma}$. Note that $\langle i, j\rangle^{\wedge} \in c_{i j}$.

2.5 Definition. Recall that we have fixed a unital $C^{*}$-algebra $A$.

(1) An $\varepsilon$-flat $\mathrm{GL}(A)$-coordinate bundle on $\Lambda$ is a collection of continuous functions $\mathbf{v}=\left\{v_{i j}: c_{i j} \rightarrow\right.$ $\left.\mathrm{GL}(A) \mid\langle i, j\rangle \in \Lambda^{(1)}\right\}$ satisfying:

(a) $v_{i j}(x) \in \mathrm{U}(A)_{\varepsilon}$ for all $x \in c_{i j}$ and all $\langle i, j\rangle \in \Lambda^{(1)}$;

(b) $v_{i j}(x)=v_{j i}(x)^{-1}$ for all $x \in c_{i j}$ and all $\langle i, j\rangle \in \Lambda^{(1)}$;

(c) $v_{i k}(x)=v_{i j}(x) v_{j k}(x)$ for all $x \in c_{i j k}$ and all $\langle i, j, k\rangle \in \Lambda^{(2)}$; and

(d) $\left\|v_{i j}(x)-v_{i j}(y)\right\|<\varepsilon$ for all $x, y \in c_{i j}$ and all $\langle i, j\rangle \in \Lambda^{(1)}$. 
(2) Define a metric $d$ on the set of all GL(A)-coordinate bundles on $\Lambda$ by

$$
d\left(\mathbf{v}, \mathbf{v}^{\prime}\right)=\max _{\langle i, j\rangle \in \Lambda} \max _{x \in c_{i j}}\left\|v_{i j}(x)-v_{i j}^{\prime}(x)\right\| .
$$

We may sometimes refer to an $\varepsilon$-flat coordinate bundle as an "almost flat coordinate bundle" without specifying $\varepsilon$. We think of an almost flat coordinate bundle $\mathbf{v}$ as a collection of transition functions defining a bundle over $|\Lambda|$, with fiber $A$, that has "small" curvature. We substantiate this point of view in Section 7 where we show that there are positive numbers $\varepsilon_{0}, \nu, r$ that depend only on $\Lambda$ such that for any $\varepsilon$-flat $\operatorname{GL}(A)$-coordinate bundle $v_{i j}$ on $\Lambda$, with $\varepsilon<\varepsilon_{0}$, there is a 1-Cech cocycle $\widetilde{v}_{i j}: V_{i} \cap V_{j} \rightarrow U(A)_{r \varepsilon}$ that extends $v_{i j}$ to prescribed open sets and $\widetilde{v}_{i j}$ is $r \varepsilon$-flat in the sense that $\left\|\widetilde{v}_{i j}(x)-\widetilde{v}_{i j}\left(x^{\prime}\right)\right\|<r \varepsilon$ for all $x \in V_{i} \cap V_{j}$. Here $V_{i}=\left\{x \in|\Lambda|: \operatorname{dist}\left(x, c_{i}\right)<\nu\right\}$. Since the sets $V_{i}$ are open, the usual gluing construction based on $\widetilde{v}_{i j}$ defines a locally trivial (almost flat) bundle. These objects are closely related to almost flat $K$-theory classes; see Section 8 for details.

\section{The correspondence between almost flat bundles and quasi- representations}

We state the main results on this topic. The proofs are given in subsequent sections.

3.1 Theorem. Let $\Lambda$ be a finite connected simplicial complex with fundamental group $\Gamma$. There exist positive numbers $C_{0}, \delta_{0}$, and $\varepsilon_{0}$ such that the following holds.

If $A$ is a unital $C^{*}$-algebra, then there are functions

$$
\left\{\begin{array}{c}
\varepsilon_{0} \text {-flat } \mathrm{GL}(A) \\
\text { coordinate bundles on } \Lambda
\end{array}\right\} \stackrel{\alpha}{\longleftarrow} \underset{\beta}{\longleftrightarrow}\left\{\begin{array}{c}
\left(\mathcal{F}_{\Lambda}, \delta_{0}\right) \text {-representations } \\
\text { of } \Gamma \text { to } \operatorname{GL}(A)
\end{array}\right\}
$$

such that:

(1) if $0<\varepsilon<\varepsilon_{0}$ and $\mathbf{v}$ is an $\varepsilon$-flat $\mathrm{GL}(A)$-coordinate bundle on $\Lambda$, then $\alpha(\mathbf{v})$ is an $\left(\mathcal{F}_{\Lambda}, C_{0} \varepsilon\right)$ representation of $\Gamma$ to $\mathrm{GL}(A)$; and

(2) if $0<\delta<\delta_{0}$ and $\pi: \Gamma \rightarrow \operatorname{GL}(A)$ is an $\left(\mathcal{F}_{\Lambda}, \delta\right)$-representation, then $\beta(\pi)$ is a $C_{0} \delta$-flat $\mathrm{GL}(A)$ coordinate bundle on $\Lambda$.

\section{Moreover:}

(3) if $0<\varepsilon<\varepsilon_{0}$ and $\mathbf{v}$ and $\mathbf{v}^{\prime}$ are $\varepsilon$-flat $\mathrm{GL}(A)$-coordinate bundles, then $d\left(\alpha(\mathbf{v}), \alpha\left(\mathbf{v}^{\prime}\right)\right)<$ $d\left(\mathbf{v}, \mathbf{v}^{\prime}\right)+C_{0} \varepsilon$.

(4) if $0<\delta<\delta_{0}$ and $\pi, \pi^{\prime}: \Gamma \rightarrow \mathrm{GL}(A)$ are $\left(\mathcal{F}_{\Lambda}, \delta\right)$-representations, then $d\left(\beta(\pi), \beta\left(\pi^{\prime}\right)\right)<$ $d\left(\pi, \pi^{\prime}\right)+C_{0} \delta$.

3.2 Definition. A GL $(A)$-coordinate bundle $\mathbf{v}=\left\{v_{i j}\right\}$ is normalized if $v_{i j}\left(\langle i, j\rangle^{\wedge}\right)=1_{A}$ for every edge $\langle i, j\rangle$ of $T$.

By Proposition 4.8 , if a vector bundle over $|\Lambda|$ may be represented by an $\varepsilon$-flat GL $(A)$-coordinate bundle, then it can be represented by a normalized $C \varepsilon$-flat $\mathrm{GL}(A)$-coordinate bundle (where $C>0$ depends only on $\Lambda$ ). 
3.3 Theorem. Let $\Lambda$ be a finite connected simplicial complex with fundamental group $\Gamma$. There exist positive numbers $C_{1}, \varepsilon_{1}$ and $\delta_{1}$ such that the following holds for any unital $C^{*}$-algebra $A$.

(1) If $0<\varepsilon<\varepsilon_{1}$ and $\mathbf{v}$ is a normalized $\varepsilon$-flat $\mathrm{GL}(A)$-coordinate bundle on $\Lambda$, then

$$
d((\beta \circ \alpha)(\mathbf{v}), \mathbf{v}) \leq C_{1} \varepsilon .
$$

(2) If $0<\delta<\delta_{1}$ and $\pi: \Gamma \rightarrow \mathrm{GL}(A)$ is an $\left(\mathcal{F}_{\Lambda}, \delta\right)$-representation, then

$$
d((\alpha \circ \beta)(\pi), \pi) \leq C_{1} \delta .
$$

\section{From almost flat bundles to quasi-representations}

In this section we construct the map $\alpha$ announced in Section 3. It is a combinatorial version of a construction due to Connes-Gromov-Moscovici [3] involving parallel transport on a smooth manifold.

Let $\mathbf{v}=\left\{v_{i j}: c_{i j} \rightarrow \mathrm{GL}(A)\right\}$ be an $\varepsilon$-flat coordinate bundle on $\Lambda$. We will define a quasirepresentation $\alpha(\mathbf{v}): \Gamma \rightarrow \mathrm{GL}(A)$ with properties described in Proposition 4.6 .

4.1 Notation. Recall that the barycenter of a 1 -simplex $\langle i, j\rangle \in \Lambda^{(1)}$ is written $\langle i, j\rangle^{\wedge}$. For such a 1-simplex, let $\stackrel{\circ}{i j}_{i j}=v_{i j}\left(\langle i, j\rangle^{\wedge}\right) \in \mathrm{GL}(A)$. For a path $I=\left(i_{1}, \ldots, i_{m}\right)$ of vertices in $\Lambda$, let

$$
\stackrel{\circ}{v}_{I}={\stackrel{\circ}{i_{1} i_{2}}}_{2} \ldots{\stackrel{\circ}{i_{m-1} i_{m}}}
$$

4.2 Definition. Define a group homomorphism $\tilde{\pi}=\tilde{\pi}_{\mathbf{v}}: \mathbb{F}_{\Lambda} \rightarrow \operatorname{GL}(A)$ as follows. If $\langle i, j\rangle \in \Lambda^{(1)}$, let $I=\left(i_{0}, \ldots, i\right)$ be the unique path along $T$ from $i_{0}$ to $i$ and $J=\left(i_{0}, \ldots, j\right)$ be the unique path from $i_{0}$ to $j$. Set

$$
\tilde{\pi}(\langle i, j\rangle)=\stackrel{\circ}{v}_{I} \stackrel{\circ}{i j}_{i j} \stackrel{\circ}{J}^{-1}
$$

Finally, set

$$
\alpha(\mathbf{v})=\tilde{\pi} \circ s: \Gamma \rightarrow \operatorname{GL}(A),
$$

where $s$ is the set theoretic section of $q: \mathbb{F}_{\Lambda} \rightarrow \Gamma$ that was fixed in Notation 2.1.

4.3 Lemma. Let $\nu>0$ and $0<\varepsilon<1$. If $x_{1}, \ldots, x_{m} \in A, u_{1}, \ldots, u_{m} \in \mathrm{U}(A)$ and $\left\|x_{i}-u_{i}\right\|<\nu \varepsilon$ for all $i$, then $\left\|x_{1} \ldots x_{m}-u_{1} \ldots u_{m}\right\|<(1+\nu)^{m} \varepsilon$. In particular, if $x_{1}, \ldots, x_{m} \in \mathrm{U}(A)_{\varepsilon}$, then $x_{1} \ldots x_{m} \in \mathrm{U}(A)_{2^{m}}$.

Proof. For $i \in\{1, \ldots, m\}$, let $u_{i} \in \mathrm{U}(A)$ be such that $\left\|x_{i}-u_{i}\right\|<\varepsilon$. One checks that

$$
\left\|x_{1} \ldots x_{m}-u_{1} \ldots u_{m}\right\|<\sum_{i=1}^{m}\left\|x_{i}-u_{i}\right\|(1+\nu \varepsilon)^{m-i}<(1+\nu)^{m} \varepsilon .
$$

4.4 Notation. For $g \in \mathbb{F}_{\Lambda}$, let $\ell(g) \in \mathbb{Z}_{\geq 0}$ be the word length of $g$ with respect to the generating set $\Lambda^{(1)}$. We denote by $L$ the length (number of edges) of a longest path in $\Lambda$ that starts at the root $i_{0}$ and does not repeat any edge.

4.5 Lemma. If $g \in \mathbb{F}_{\Lambda}$, then $\operatorname{dist}(\tilde{\pi}(g), \mathrm{U}(A))<2^{3 L+\ell(g)} \varepsilon$.

Proof. Equation (4.1) implies that $\tilde{\pi}(\langle i, j\rangle)$ is a product of at most $3 L$ elements of $\mathrm{U}(A)_{\varepsilon}$ for any edge $\langle i, j\rangle$ of $\Lambda$. It follows from Lemma 4.3 that $\operatorname{dist}(\tilde{\pi}(\langle i, j\rangle), \mathrm{U}(A))<2^{3 L} \varepsilon$. Another application of Lemma 4.3 ends the proof. 
4.6 Proposition. There is a constant $C_{0}^{\prime}>0$, depending only on $\Lambda, T, i_{0}$, and $s$, such that if $\mathbf{v}$ is an $\varepsilon$-flat $\mathrm{GL}(A)$-coordinate bundle on $\Lambda$, then $\alpha(\mathbf{v})$ is an $\left(\mathcal{F}_{\Lambda}, C_{0}^{\prime} \varepsilon\right)$-representation of $\Gamma$ on $\operatorname{GL}(A)$.

Proof. Write $\pi:=\alpha(\mathbf{v})$. First we define a few constants so the proof will run more smoothly.

Let $\ell_{0}=\max \left\{\ell(s(\gamma)): \gamma \in \mathcal{F}_{\Lambda} \cup \mathcal{F}_{\Lambda} \cdot \mathcal{F}_{\Lambda}\right\}$.

If $\gamma, \gamma^{\prime} \in \Gamma$, then $s(\gamma) s\left(\gamma^{\prime}\right) s\left(\gamma \gamma^{\prime}\right)^{-1}$ belongs to the kernel of $q: \mathbb{F}_{\Lambda} \rightarrow \Gamma$, that is, to the normal subgroup generated by the set of relators $\mathcal{R}$. (See Notation 2.1.) For each pair $\gamma, \gamma^{\prime} \in \mathcal{F}_{\Lambda}$ choose and fix a representation

$$
s(\gamma) s\left(\gamma^{\prime}\right) s\left(\gamma \gamma^{\prime}\right)^{-1}=\prod_{n=1}^{m\left(\gamma, \gamma^{\prime}\right)} x_{n} r_{n} x_{n}^{-1}
$$

with $\left\{x_{n}\right\} \subset \mathbb{F}_{\Lambda}$ and $\left\{r_{n}\right\} \subset \mathcal{R}$. Let $m=\max \left\{m\left(\gamma, \gamma^{\prime}\right): \gamma, \gamma^{\prime} \in \mathcal{F}_{\Lambda}\right\}$ and let $\ell_{1}$ be the maximum of the lengths $\ell\left(x_{n}\right)$ of all the elements $x_{n}$ that appear in equation 4.2 for all pairs $\gamma, \gamma^{\prime} \in \mathcal{F}_{\Lambda}$. Finally, let $C_{0}^{\prime}=2^{\left(15 L+2 \ell_{1}\right) m} \cdot 2^{4 L+\ell_{0}}$.

For any $\gamma, \gamma^{\prime} \in \mathcal{F}_{\Lambda}$, we show that $\left\|\pi(\gamma) \pi\left(\gamma^{\prime}\right)-\pi\left(\gamma \gamma^{\prime}\right)\right\|<C_{0}^{\prime} \varepsilon$. Using Lemma 4.5 we note that $\left\|\tilde{\pi}\left(s\left(\gamma \gamma^{\prime}\right)\right)\right\|<1+2^{3 L+\ell_{0}} \varepsilon<2^{4 L+\ell_{0}}$ and hence

$$
\begin{aligned}
\left\|\pi(\gamma) \pi\left(\gamma^{\prime}\right)-\pi\left(\gamma \gamma^{\prime}\right)\right\| & \leq\left\|\tilde{\pi}\left(s(\gamma) s\left(\gamma^{\prime}\right) s\left(\gamma \gamma^{\prime}\right)^{-1}\right)-1\right\|\left\|\tilde{\pi}\left(s\left(\gamma \gamma^{\prime}\right)\right)\right\| \\
& \leq 2^{4 t+\ell_{0}}\left\|\tilde{\pi}\left(s(\gamma) s\left(\gamma^{\prime}\right) s\left(\gamma \gamma^{\prime}\right)^{-1}\right)-1\right\| .
\end{aligned}
$$

Now, $r_{n} \in \mathcal{R}$ implies that either $r_{n}=\langle i, j\rangle$ is an edge of $T$ in which case $\tilde{\pi}\left(r_{n}\right)=1$ or $r_{n}=\langle i, j\rangle\langle j, k\rangle\langle i, k\rangle^{-1}$ for some vertices $i, j, k$, and hence

$$
\tilde{\pi}\left(r_{n}\right)=\stackrel{\circ}{v}_{I} \stackrel{\circ}{v}_{i j} \stackrel{\circ}{J}_{J}^{-1} \cdot \stackrel{\circ}{v}_{J} \stackrel{\circ}{j k}_{j k} \stackrel{\circ}{K}_{K}^{-1} \cdot \stackrel{\circ}{v}_{K} \stackrel{\circ}{v}_{i k}^{-1} \stackrel{\circ}{v}_{I}^{-1}=\stackrel{\circ}{v}_{I} \cdot \stackrel{\circ}{i j}_{i j} \stackrel{\circ}{j k}_{j} \stackrel{\circ}{i k}_{i k}^{-1} \cdot \stackrel{\circ}{v}_{I}^{-1}
$$

Let $t$ be the barycenter $\langle i, j, k\rangle^{\wedge}$. Since $v_{i j}, v_{j k}$, and $v_{i k}^{-1}$ are $\varepsilon$-constant with norm $\leq 1+\varepsilon$, we get that

$$
\begin{aligned}
\left\|\tilde{\pi}\left(r_{n}\right)-1\right\| & \leq\left\|\stackrel{\circ}{v}_{I} \cdot \stackrel{\circ}{v}_{i j} \stackrel{\circ}{v}_{j k} \stackrel{\circ}{v}_{i k}^{-1} \cdot \stackrel{\circ}{v}_{I}^{-1}-\stackrel{\circ}{v}_{I} \cdot v_{i j}(t) v_{j k}(t) v_{i k}^{-1}(t) \cdot \stackrel{\circ}{v}_{I}^{-1}\right\| \\
& <\left\|\stackrel{\circ}{v}_{I}\right\|\left\|\stackrel{\circ}{I}_{I}^{-1}\right\|(1+\varepsilon)^{2} 3 \varepsilon \\
& <2^{2 L+4} \varepsilon \leq 2^{6 L} \varepsilon .
\end{aligned}
$$

By Lemma $4.5\left\|\tilde{\pi}\left(x_{n}\right)\right\|,\left\|\tilde{\pi}\left(x_{n}^{-1}\right)\right\| \leq 1+2^{3 L+\ell\left(x_{n}\right)} \varepsilon<2^{4 L+\ell_{1}}$. Therefore

$$
\left\|\tilde{\pi}\left(x_{n}\right) \tilde{\pi}\left(r_{n}\right) \tilde{\pi}\left(x_{n}^{-1}\right)-1\right\|<\left\|\tilde{\pi}\left(x_{n}\right)\right\|\left\|\tilde{\pi}\left(x_{n}^{-1}\right)\right\|\left\|\tilde{\pi}\left(r_{n}\right)-1\right\| \leq 2^{14 L+2 \ell_{1}} \varepsilon .
$$

Because

$$
\tilde{\pi}\left(s(\gamma) s\left(\gamma^{\prime}\right) s\left(\gamma \gamma^{\prime}\right)^{-1}\right)=\prod_{n=1}^{m\left(\gamma, \gamma^{\prime}\right)} \tilde{\pi}\left(x_{n}\right) \tilde{\pi}\left(r_{n}\right) \tilde{\pi}\left(x_{n}^{-1}\right)
$$

applying Lemma 4.5 again we get

$$
\left\|\tilde{\pi}\left(s(\gamma) s\left(\gamma^{\prime}\right) s\left(\gamma \gamma^{\prime}\right)^{-1}\right)-1\right\|<\left(1+2^{14 L+2 \ell_{1}}\right)^{m} \varepsilon \leq 2^{\left(15 L+2 \ell_{1}\right) m} \varepsilon .
$$

Combined with (4.3), this proves that for all $\gamma, \gamma^{\prime} \in \mathcal{F}_{\Lambda}$

$$
\left\|\pi(\gamma) \pi\left(\gamma^{\prime}\right)-\pi\left(\gamma \gamma^{\prime}\right)\right\|<2^{4 L+\ell_{0}} \cdot 2^{\left(15 L+2 \ell_{1}\right) m} \varepsilon=C_{0}^{\prime} \varepsilon .
$$

We must also prove that $\pi(\gamma) \in \mathrm{U}(A)_{C_{0}^{\prime} \varepsilon}$ if $\gamma \in \mathcal{F}_{\Lambda}$. This follows immediately from Lemma 4.5 since $\pi(\gamma)=\tilde{\pi}(s(\gamma))$ and $\ell(\gamma) \leq \ell_{0}$. 
Let us single out an estimate from the proof for later use (cf. (4.4)).

4.7 Lemma. There exists $K>0$, depending only on $\Lambda, T, i_{0}$, and $s$, such that the following holds.

Suppose $\mathbf{v}$ is an $\varepsilon$-flat $\mathrm{GL}(A)$-coordinate bundle on $\Lambda$. Let $\tilde{\pi}=\tilde{\pi}_{\mathbf{v}}$ be as in Definition 4.2. If $\langle i, j\rangle \in \Lambda^{(1)}$, then $g_{i j}:=s\left(\gamma_{i j}\right) \cdot\langle i, j\rangle^{-1} \in \operatorname{ker} q$ and $\left\|\tilde{\pi}\left(g_{i j}\right)-1_{A}\right\|<K \varepsilon$.

4.8 Proposition. Suppose $\mathbf{v}=\left\{v_{i j}\right\}$ is an $\varepsilon$-flat $\mathrm{GL}(A)$-coordinate bundle on $\Lambda$. Then there exist a constant $C>0$, depending only on $\Lambda$, and elements $\lambda_{i} \in \mathrm{GL}(A)$ such that the coordinate bundle $\mathbf{w}=\left\{w_{i j}\right\}$ defined by $w_{i j}=\lambda_{i} v_{i j} \lambda_{j}^{-1}$ is normalized and $C \varepsilon$-flat; $\mathbf{v}$ and $\mathbf{w}$ yield isomorphic bundles on $|\Lambda|$.

Proof. Recall that $L$ is the length of the longest path in $T$ that starts at the root $i_{0}$ and has no backtracking. We show that $C:=4^{L+1}$ verifies the statement. For each vertex $i$ of $\Lambda$, let $I=\left(i_{0}, \ldots, i\right)$ be the unique path along $T$ from $i_{0}$ to $i$ and using notation as in 4.1 and 4.2 set $\lambda_{i}:=\stackrel{\circ}{v}_{I}$. Thus $w_{i j}=\dot{o}_{I} v_{i j} \stackrel{\circ}{J}_{J}^{-1}$.

Then clearly $w_{i j}\left(\langle i, j\rangle^{\wedge}\right)=1_{A}$ for all $\langle i, j\rangle \in T^{(1)}$. Moreover $\left\|\lambda_{i}\right\|,\left\|\lambda_{i}^{-1}\right\|<(1+\varepsilon)^{L}<2^{L}$ since $\| \stackrel{\circ}{i, j}_{i, j}<1+\varepsilon$ by hypothesis. If $\langle i, j\rangle$ is a 1-simplex of $\Lambda$ and $x, y \in c_{i j}$, then

$$
\left\|w_{i j}(x)-w_{i j}(y)\right\|=\left\|\lambda_{i}\left(v_{i j}(x)-v_{i j}(y)\right) \lambda_{j}^{-1}\right\|<2^{L} \cdot \varepsilon \cdot 2^{L}<C \varepsilon .
$$

Moreover, since $\lambda_{i}, \lambda_{j}^{-1} \in \mathrm{U}(A)_{2{ }^{L} \varepsilon}$ by Lemma 4.3 and $v_{i j}(x) \in \mathrm{U}(A)_{\varepsilon}$ it follows immediately that $w_{i j}(x) \in \mathrm{U}(A)_{4}{ }^{L+1} \varepsilon$. Therefore, $\mathbf{w}$ is $C \varepsilon$-flat.

By [12, Theorem 3.2] $\mathbf{v}$ and $\mathbf{w}$ yield isomorphic bundles on $|\Lambda|$.

\section{From quasi-representations to almost flat bundles}

In this section we describe the map $\beta$ announced in Section 3 . The idea is to extend a quasirepresentation of $\Gamma$ to a quasi-representation of the fundamental groupoid of $\Lambda$ and reinterpret the latter as a lattice gauge field. This enables us to invoke a construction of Phillips and Stone [19,20] that associates an almost flat coordinate bundle to a lattice gauge field with controlled distortion and small modulus of continuity. For the sake of completeness we give a full account of this construction.

Let $0<\delta<1 / 140 L$, with $L$ as in 4.4 , and let an $\left(\mathcal{F}_{\Lambda}, \delta\right)$-representation $\pi$ of $\Gamma$ be given. We will define an almost flat coordinate bundle $\beta(\pi)$ on $\Lambda$.

The following notation will be used in the definition.

5.1 Notation. We fix a partial order $\mathbf{o}$ on the vertices of $\Lambda$ such that the set of vertices of any simplex of $\Lambda$ is a totally ordered set under o. One may always assume that such an order exists by passing to the first barycentric subdivision of $\Lambda$ : if $\hat{\sigma}_{1}$ and $\hat{\sigma}_{2}$ are the barycenters of simplices $\sigma_{1}$ and $\sigma_{2}$ of $\Lambda$, define $\hat{\sigma}_{1}<\hat{\sigma}_{2}$ if $\sigma_{1}$ is a face of $\sigma_{2}$ (cf. [20]).

When we write $\sigma=\left\langle i_{1}, \ldots, i_{m}\right\rangle$ it is implicit that the vertices of $\sigma$ are written in increasing o-order.

5.2 Notation. Following [20], we re-parametrize the dual cell blocks $c_{i}^{\sigma}$ using "modified barycentric coordinates" $\left(s_{0}, \ldots, s_{r}\right)$. These are defined in terms of the barycentric coordinates by $s_{j}=t_{j} / t_{i}$. In these coordinates $c_{i}^{\sigma}$ is identified with the cube

$$
\left\{\left(s_{0}, \ldots, s_{i}, \ldots, s_{r}\right) \mid s_{i}=1 \text { and } 0 \leq s_{j} \leq 1 \text { for all } j \neq i\right\} .
$$

See Figure 2 . 


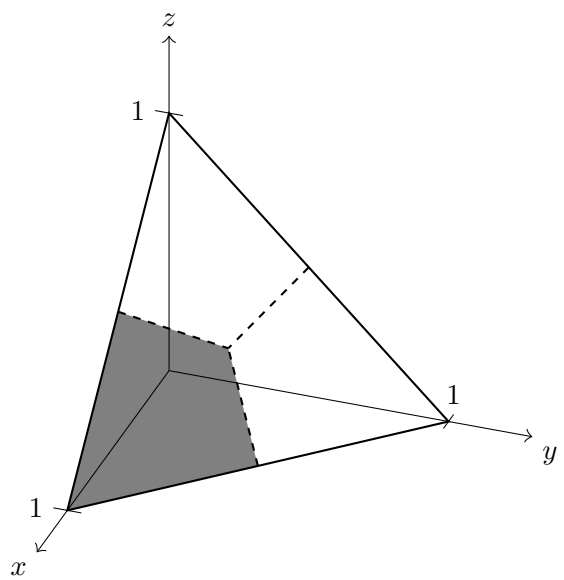

(a)

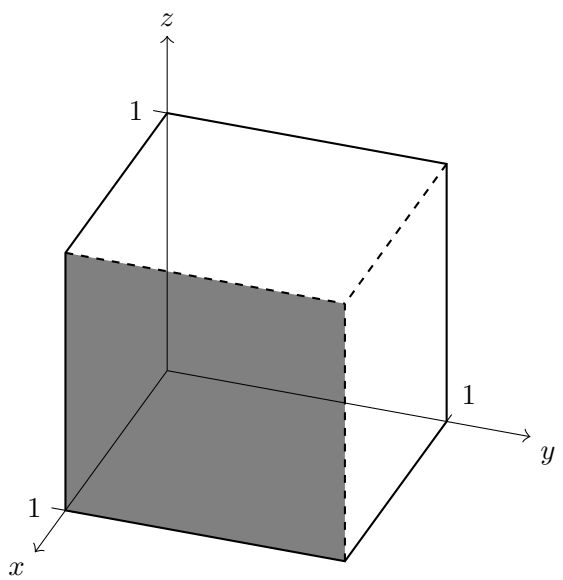

(b)

Figure 2: (a) The identification of a dual cell block in a 2-simplex as given by barycentric coordinates. (b) The identification of a dual cell block in a 2-simplex as given by modified barycentric coordinates.

The construction of $\beta(\pi)$ is inspired by (and borrows heavily from) the work of Phillips and Stone [20]. It is somewhat involved, but we outline the procedure in the following definition before going into the details.

5.3 Definition. Let $i$ and $j$ be adjacent vertices of $\Lambda$. We will define $\beta(\pi)=\left\{v_{i j}: c_{i j} \rightarrow \operatorname{GL}(A)\right\}$ by defining $v_{i j}$ on all the dual cell blocks $c_{i j}^{\sigma}$ such that $\sigma$ contains $i$ and $j$.

(1) Let $u_{i j}:=\breve{\pi}\left(\gamma_{i j}\right)$, where $\breve{\pi}: \Gamma \rightarrow \mathrm{U}(A)$ is the perturbation of $\pi$ provided by Proposition 5.6.

(2) Suppose $\sigma$ is a simplex in $\Lambda$ containing $i$ and $j$ and that $i<j$. Write $\sigma$ (in increasing o-order) as $\sigma=\langle 0, \ldots, i, \ldots, j, \ldots, r\rangle$.

For an o-ordered subset of vertices $I=\left\{i=i_{1}<i_{2}<i_{3}<\cdots<i_{m}=j\right\}$, set

$$
u_{I}:=u_{i_{1} i_{2}} u_{i_{2} i_{3}} \ldots u_{i_{m-1} i_{m}}
$$

(where it is understood that if $I=\{i<j\}$, then $u_{I}=u_{i j}$ ).

(3) Define $v_{i j}^{\sigma}: c_{i j}^{\sigma} \rightarrow A$, using modified barycentric coordinates on $c_{i j}^{\sigma}$ (see 5.2 , as follows. For $\mathbf{s}=\left(s_{0}, \ldots, s_{i}=1, \ldots, s_{j}=1, \ldots, s_{r}\right) \in c_{i j}^{\sigma}$ let

$$
v_{i j}^{\sigma}(\mathbf{s}):=\sum_{I} \lambda_{I}(\mathbf{s}) u_{I},
$$

where

$$
\lambda_{I}(\mathbf{s})=\lambda_{I}^{\sigma}(\mathbf{s})=\prod_{i \leq k \leq j} s_{k}^{\prime}
$$

with $s_{k}^{\prime}=s_{k}$ if $k \in I$ and $s_{k}^{\prime}=1-s_{k}$ if $k \notin I$.

The sum above is over the subsets $I$ of $\{i, \ldots, j\} \subseteq \sigma^{(0)}$ that contain both $i$ and $j$ as above. One can identify the subsets $I$ with ascending paths from $i$ to $j$ that are contained in $\sigma$. Let us note that $\sum_{I} \lambda_{I}(\mathbf{s})=1$ for $\mathbf{s} \in c_{i j}^{\sigma}$ since $\prod_{i<k<j}\left(\left(s_{k}+\left(1-s_{k}\right)\right)=1\right.$. We will see that the range of $v_{i j}^{\sigma}$ is actually contained in $\operatorname{GL}(A)$. If $i>j$, let $v_{i j}^{\sigma}$ be the pointwise inverse of $v_{j i}^{\sigma}$. 
(4) Corollary 5.10 below shows that for each $\langle i, j\rangle \in \Lambda^{(1)}$ the collection of all $v_{i j}^{\sigma}$ above determines a function $v_{i j}: c_{i j} \rightarrow \mathrm{GL}(A)$. We define $\beta(\pi):=\left\{v_{i j}\right\}$.

5.4 Proposition. There exist positive numbers $C_{0}^{\prime \prime}$ and $\delta_{0}$, depending only on $\Lambda$, such that if $0<\delta<\delta_{0}$ and $\pi: \Gamma \rightarrow \mathrm{GL}(A)$ is an $\left(\mathcal{F}_{\Lambda}, \delta\right)$-representation of $\Gamma$, then $\beta(\pi)$ is a $C_{0}^{\prime \prime} \delta$-flat $\mathrm{GL}(A)$ coordinate bundle on $\Lambda$.

The rest of the section is devoted to the proof of Proposition 5.4.

5.5 Remark. The construction described in Definition 5.3 is an attempt to have $v_{i j}$ be "as constant as possible" and equal to $u_{i j}$ at the barycenter of $\langle i, j\rangle$ (cf. [19, Sec. 2]). The cocycle condition one might hope for would force relations of the form $u_{i j} u_{j k}=u_{i k}$, which do not necessarily hold since $\pi$ (and therefore $\breve{\pi}$ ) is only approximately multiplicative. The definition of $v_{i j}^{\sigma}$ uses successive linear interpolation to account for this. For example:

(1) If $\sigma=\langle 0,1,2\rangle$, then $v_{01}^{\sigma}=u_{01}$ and $v_{12}^{\sigma}=u_{12}$, but

$$
v_{02}^{\sigma}\left(s_{0}=1, s_{1}, s_{2}=1\right)=s_{1} u_{01} u_{12}+\left(1-s_{1}\right) u_{02} .
$$

(2) If $\sigma=\langle 0,1,2,3\rangle$, then $v_{01}^{\sigma}=u_{01}, v_{12}^{\sigma}=u_{12}, v_{23}^{\sigma}=u_{23}$,

$$
\begin{aligned}
& v_{02}^{\sigma}\left(s_{0}=1, s_{1}, s_{2}=1\right)=s_{1} u_{01} u_{12}+\left(1-s_{1}\right) u_{02}, \\
& v_{13}^{\sigma}\left(s_{1}=1, s_{2}, s_{3}=1\right)=s_{2} u_{12} u_{23}+\left(1-s_{2}\right) u_{13},
\end{aligned}
$$

and

$$
\begin{aligned}
v_{03}^{\sigma}\left(s_{0}=1, s_{1}, s_{2}, s_{3}=1\right)= & s_{1} s_{2} u_{01} u_{12} u_{13}+s_{1}\left(1-s_{2}\right) u_{01} u_{13}+ \\
& +\left(1-s_{1}\right) s_{2} u_{02} u_{23}+\left(1-s_{1}\right)\left(1-s_{2}\right) u_{03} .
\end{aligned}
$$

Notice that in the modified barycentric coordinates $\left(s_{0}, \ldots, s_{r}\right)$ the barycenter of $\langle i, j\rangle$ is given by $s_{i}=s_{j}=1$ and $s_{k}=0$ for all $k \notin\{i, j\}$. Therefore, $v_{i j}^{\sigma}\left(\langle i, j\rangle^{\wedge}\right)=u_{i j}$ as desired.

To start the construction, we first perturb $\pi$ slightly so that we can deal with unitary elements instead of just invertible ones when convenient.

5.6 Proposition. Given an $\left(\mathcal{F}_{\Lambda}, \delta\right)$-representation $\pi: \Gamma \rightarrow \mathrm{GL}(A), 0<\delta<1 / 7$, there exists a function $\breve{\pi}: \Gamma \rightarrow \mathrm{U}(A)$ such that

(1) $\breve{\pi}(e)=1_{A}$;

(2) $\breve{\pi}(\gamma) \in \mathrm{U}(A)$ for all $\gamma \in \mathcal{F}_{\Lambda}$;

(3) $\breve{\pi}\left(\gamma^{-1}\right)=\breve{\pi}(\gamma)^{*}$ for all $\gamma \in \mathcal{F}_{\Lambda}$;

(4) $\left\|\breve{\pi}\left(\gamma \gamma^{\prime}\right)-\breve{\pi}(\gamma) \breve{\pi}\left(\gamma^{\prime}\right)\right\|<70 \delta$ for all $\gamma, \gamma^{\prime} \in \mathcal{F}_{\Lambda}$ with $\gamma \gamma^{\prime} \in \mathcal{F}_{\Lambda}$; and

(5) $\|\breve{\pi}(\gamma)-\pi(\gamma)\|<20 \delta$ for all $\gamma \in \mathcal{F}_{\Lambda}$.

The next lemma will be used in the proof.

5.7 Lemma. Let $\omega: \mathrm{GL}(A) \rightarrow \mathrm{U}(A)$ be given by $\omega(v)=v\left(v^{*} v\right)^{-1 / 2}$. If $v \in \mathrm{U}(A)_{\delta}, 0<\delta<1 / 7$, then $\|\omega(v)-v\|<5 \delta$. 
Proof. Observe that for $z \in A$

$$
\|z-1\| \leq \theta<1 / 2 \quad \Rightarrow \quad\left\|z^{-1}-1\right\|<2 \theta,
$$

using the Neumann series.

Assume $v \in \mathrm{U}(A)_{\delta}$. Then there is $u \in \mathrm{U}(A)$ such that $\|v-u\|<\delta$, so $\left\|v u^{*}-1\right\|<\delta$. Let $w=v u^{*}$. Then

$$
\omega(w)=v u^{*}\left(u v^{*} v u^{*}\right)^{-1 / 2}=v u^{*} u\left(v^{*} v\right)^{-1 / 2} u^{*}=\omega(v) u^{*}
$$

and thus $\|\omega(w)-w\|=\|\omega(v)-v\|$.

Now $\|w\|<1+\delta$, so $\left\|w^{*} w-1\right\| \leq\left\|\left(w^{*}-1\right) w\right\|+\|w-1\|<\delta(1+\delta)+\delta<15 \delta / 7$. Therefore, by (5.1), $\left\|\left(w^{*} w\right)^{-1 / 2}-1\right\| \leq\left\|\left(w^{*} w\right)^{-1}-1\right\|<30 \delta / 7$. Finally,

$$
\|\omega(v)-v\|=\left\|w\left(w^{*} w\right)^{-1 / 2}-w\right\|<(1+\delta) 30 \delta / 7<5 \delta .
$$

Proof of Proposition 5.6. The idea is simple: define $\breve{\pi}=\omega \circ \sigma$ where $\omega$ is as in Lemma 5.7 and $\sigma: \Gamma \rightarrow A$ is the function

$$
\sigma(\gamma)=\frac{\pi(\gamma)+\pi\left(\gamma^{-1}\right)^{*}}{2}
$$

We check the required properties.

Let $\gamma \in \mathcal{F}_{\Lambda}$. First we prove that $\left\|\pi\left(\gamma^{-1}\right)-\pi(\gamma)^{-1}\right\|<2 \delta$ and $\left\|\pi(\gamma)^{-1}-\pi(\gamma)^{*}\right\|<3 \delta$.

Because $\pi(\gamma) \in \mathrm{U}(A)_{\delta}$, we can write $\pi(\gamma)=u v$ for some $u \in \mathrm{U}(A)$ and $v \in \mathrm{GL}(A)$ with $\|v-1\|<\delta$. Then $\left\|\pi(\gamma)^{-1}-u^{*}\right\|=\left\|v^{-1}-1\right\|<2 \delta$ by (5.1) and hence $\left\|\pi(\gamma)^{-1}\right\|<1+2 \delta$. Therefore,

$$
\begin{aligned}
\left\|\pi\left(\gamma^{-1}\right)-\pi(\gamma)^{-1}\right\| & =\left\|\left(\pi\left(\gamma^{-1}\right) \pi(\gamma)-1\right) \pi(\gamma)^{-1}\right\| \\
& \leq\left\|\pi\left(\gamma^{-1}\right) \pi(\gamma)-\pi\left(\gamma^{-1} \gamma\right)\right\|\left\|\pi(\gamma)^{-1}\right\| \\
& \leq \delta(1+2 \delta)<2 \delta .
\end{aligned}
$$

It is just as plain to see that

$$
\left\|\pi(\gamma)^{-1}-\pi(\gamma)^{*}\right\|=\left\|(u v)^{-1}-(u v)^{*}\right\|=\left\|v^{-1}-v^{*}\right\| \leq\left\|v^{-1}-1\right\|+\left\|v^{*}-1\right\|<3 \delta,
$$

as claimed. Using these bounds we see that

$$
\begin{aligned}
\|\sigma(\gamma)-\pi(\gamma)\| & =\frac{1}{2}\left\|\pi\left(\gamma^{-1}\right)^{*}-\pi(\gamma)\right\|=\frac{1}{2}\left\|\pi\left(\gamma^{-1}\right)-\pi(\gamma)^{*}\right\| \\
& \leq \frac{1}{2}\left\|\pi\left(\gamma^{-1}\right)-\pi(\gamma)^{-1}\right\|+\frac{1}{2}\left\|\pi(\gamma)^{-1}-\pi(\gamma)^{*}\right\| \\
& <5 \delta / 2
\end{aligned}
$$

Thus

$$
\operatorname{dist}(\sigma(\gamma), \mathrm{U}(A))<5 \delta / 2+\operatorname{dist}(\pi(\gamma), \mathrm{U}(A))<7 \delta / 2<1 / 2 .
$$

In particular $\sigma(\gamma) \in \mathrm{GL}(A)$. Items (1) and (2) in the statement of the proposition are immediate. For (3) observe that if $z \in \operatorname{GL}(A)$, then $\omega\left(z^{*}\right)=\omega(z)^{*}$. It follows that $\breve{\pi}\left(\gamma^{-1}\right)=\omega\left(\sigma\left(\gamma^{-1}\right)\right)=$ $\omega\left(\sigma(\gamma)^{*}\right)=\omega(\sigma(\gamma))^{*}=\breve{\pi}(\gamma)^{*}$.

We deal with (5). From 5.3) and Lemma 5.7 we obtain $\|\breve{\pi}(\gamma)-\sigma(\gamma)\|<35 \delta / 2$. Together with (5.2) this gives

$$
\|\breve{\pi}(\gamma)-\pi(\gamma)\| \leq\|\breve{\pi}(\gamma)-\sigma(\gamma)\|+\|\sigma(\gamma)-\pi(\gamma)\|<20 \delta .
$$


We are left with (4). Suppose $\gamma, \gamma^{\prime} \in \mathcal{F}_{\Lambda}$ are such that $\gamma \gamma^{\prime} \in \mathcal{F}_{\Lambda}$. Then

$$
\begin{aligned}
\left\|\breve{\pi}(\gamma) \breve{\pi}\left(\gamma^{\prime}\right)-\breve{\pi}\left(\gamma \gamma^{\prime}\right)\right\| \leq & \|\breve{\pi}(\gamma)-\pi(\gamma)\|\left\|\breve{\pi}\left(\gamma^{\prime}\right)\right\|+\|\pi(\gamma)\|\left\|\breve{\pi}\left(\gamma^{\prime}\right)-\pi\left(\gamma^{\prime}\right)\right\|+ \\
& +\left\|\pi(\gamma) \pi\left(\gamma^{\prime}\right)-\pi\left(\gamma \gamma^{\prime}\right)\right\|+\left\|\breve{\pi}\left(\gamma \gamma^{\prime}\right)-\pi\left(\gamma \gamma^{\prime}\right)\right\| \\
< & 20 \delta+(1+\delta) 20 \delta+\delta+20 \delta \\
< & 70 \delta
\end{aligned}
$$

The next proposition allows us to use induction on the number of vertices of $\sigma$ in the proofs that follow.

5.8 Proposition. If $\sigma \subset \widetilde{\sigma}$ are simplices of $\Lambda$ and $i<j$ are vertices of $\sigma$, then the restriction of $v_{i j}^{\widetilde{\sigma}}$ to $c_{i j}^{\sigma}$ is equal to $v_{i j}^{\sigma}$.

Proof. We may assume that $\tilde{\sigma}=\sigma \cup\{l\}$ is a simplex of $\Lambda$ that has $\sigma$ as one of its faces and $l \notin \sigma$. If $\mathbf{s}=\left(s_{0}, \ldots, s_{l}, \ldots, s_{r}\right) \in c_{i j}^{\widetilde{\sigma}}$, then $s_{i}=s_{j}=1$ and moreover $\mathbf{s} \in c_{i j}^{\sigma}$ precisely when $s_{l}=0$. Let $I$ be a subset of $\{i, \ldots, j\}$ that contains both $i$ and $j$ as above. If either $l<i$ or $j<l$, then $\sigma$ and $\widetilde{\sigma}$ have exactly the same set of increasing paths from $i$ to $j$ and hence $v_{i j}^{\widetilde{\sigma}}(\mathbf{s})=v_{i j}^{\sigma}(\mathbf{s})$ for $\mathbf{s} \in c_{i j}^{\sigma}$ by Definition 5.3. (In fact, $v_{i j}^{\widetilde{\sigma}}\left(s_{0}, \ldots, s_{l}, \ldots, s_{r}\right)=v_{i j}^{\sigma}\left(s_{0}, \ldots, s_{l}=0, \ldots, s_{r}\right)$, again by Definition 5.3.)

Suppose now that $i<l<j$. Let $I=\left\{i=i_{0}<i_{1}<i_{2}<\cdots<i_{m}=j\right\}$ be an increasing path in $\widetilde{\sigma}$. If $l \notin I$ and $\mathbf{s} \in c_{i j}^{\sigma}=\left\{\mathbf{s} \in c_{i j}^{\widetilde{\sigma}}: s_{l}=0\right\}$, then $\lambda_{I}^{\widetilde{\sigma}}(\mathbf{s}) u_{I}=\left(1-s_{l}\right) \lambda_{I}^{\sigma}(\mathbf{s}) u_{I}=\lambda_{I}^{\sigma}(\mathbf{s}) u_{I}$. On the other hand if $l \in I$, then $\lambda_{I}^{\widetilde{\sigma}}(\mathbf{s})=0$ since $s_{l}$ is one of its factors. The statement follows now immediately from by Definition 5.3 since

$$
v_{i j}^{\widetilde{\sigma}}(\mathbf{s}):=\sum_{l \in I} \lambda_{I}^{\widetilde{\sigma}}(\mathbf{s}) u_{I}+\sum_{l \notin I} \lambda_{I}^{\widetilde{\sigma}}(\mathbf{s}) u_{I}
$$

5.9 Proposition. If $i<l<j$ are vertices of a simplex $\sigma$ of $\Lambda$, then $v_{i j}^{\sigma}(\mathbf{s})=v_{i l}^{\sigma}(\mathbf{s}) v_{l j}^{\sigma}(\mathbf{s})$ for all $\mathbf{s} \in c_{i l j}^{\sigma}=c_{i}^{\sigma} \cap c_{l}^{\sigma} \cap c_{j}^{\sigma}$.

Proof. Let $I=\left\{i=i_{0}<i_{1}<i_{2}<\cdots<i_{m}=j\right\}$ be an increasing path in $\sigma$.

If $l \notin I$, then $\lambda_{I}^{\sigma}(\mathbf{s})=0$ since $1-s_{l}=0$ is one of its factors. On the other hand, if $l \in I$, say

$$
I=\left\{i=i_{0}<\cdots<i_{k-1}<i_{k}=l<i_{k+1}<\cdots<i_{m}=j\right\}
$$

then letting

$$
I^{\prime}=\left\{i_{0}<\cdots<i_{k-1}<i_{k}\right\} \quad \text { and } \quad I^{\prime \prime}=\left\{i_{k}<i_{k+1}<\cdots<i_{m}\right\}
$$

we see that

$$
\lambda_{I}^{\sigma}(\mathbf{s}) u_{I}=\lambda_{I^{\prime}}^{\sigma}(\mathbf{s}) s_{l} \lambda_{I^{\prime \prime}}^{\sigma}(\mathbf{s}) u_{I^{\prime}} u_{I^{\prime \prime}}=\lambda_{I^{\prime}}^{\sigma}(\mathbf{s}) u_{I^{\prime}} \cdot \lambda_{I^{\prime \prime}}^{\sigma}(\mathbf{s}) u_{I^{\prime \prime}} .
$$

The statement now follows from Definition 5.3 since

$$
v_{i j}^{\sigma}(\mathbf{s}):=\sum_{l \in I} \lambda_{I}^{\sigma}(\mathbf{s}) u_{I}+\sum_{l \notin I} \lambda_{I}^{\sigma}(\mathbf{s}) u_{I} .
$$

5.10 Corollary. The family of functions $\left\{v_{i j}^{\sigma} \mid i, j \in \sigma\right\}$ yields a continous function $v_{i j}: c_{i j}=$ $\bigcup_{\sigma} c_{i j}^{\sigma} \rightarrow \mathrm{GL}(A)$ such that $v_{i l}(\mathbf{s}) v_{l j}(\mathbf{s})=v_{i j}(\mathbf{s})$ for all $\mathbf{s} \in c_{i l j}=c_{i} \cap c_{l} \cap c_{j}$.

Proof. Proposition 5.8 shows that if two simplices $\sigma$ and $\sigma^{\prime}$ contain $\{i, j\}$, then $v_{i j}^{\sigma}=v_{i j}^{\sigma^{\prime}}=v_{i j}^{\sigma \cap \sigma^{\prime}}$ on $c_{i j}^{\sigma \cap \sigma^{\prime}}$, so that $v_{i j}$ is well-defined. The cocycle condition follows from Proposition 5.9. It remains to show that $v_{i j}$ takes values in $\mathrm{GL}(A)$. This will follow from the estimate

$$
\left\|v_{i j}^{\sigma}(\mathbf{s})-u_{i j}\right\|<70 L \delta<1 / 2
$$


that we now verify.

If $I=\left\{i=i_{1}<i_{2}<i_{3}<\cdots<i_{m}=j\right\}$ and $u_{I}:=u_{i_{1} i_{2}} u_{i_{2} i_{3}} \ldots u_{i_{m-1} i_{m}}$ are as in Definition 5.3 , then show that $\left\|u_{I}-u_{i j}\right\|<70 m \delta$ by induction on $m$. This is trivial if $m=2$, since in that case $u_{I}=u_{i j}$. For the inductive step, we use the estimate $\left\|u_{i_{k} i_{k+1}} u_{i_{k+1} i_{k+2}}-u_{i_{k} i_{k+2}}\right\|<70 \delta$ proved in Proposition 5.6(4). Because $v_{i j}^{\sigma}(\mathbf{s}):=\sum_{I} \lambda_{I}^{\sigma}(\mathbf{s}) u_{I}$, the estimate 5.4 follows since $\sum_{I} \lambda_{I}^{\sigma}(\mathbf{s})=1$ for $\mathbf{s} \in c_{i j}^{\sigma}$.

Proof of Proposition 5.4. Let $\delta_{0}=1 / 140 L$.

Corollary 5.10 all but implies Proposition 5.4. To complete the proof, we need to verify the almost flatness condition. Assume $i<j$ are vertices as in the proof of Corollary 5.10, We have seen that $\left\|v_{i j}(x)-u_{i j}\right\|<70 L \delta$ for all $x \in c_{i j}$. Since $u_{i j}$ is a unitary and since $0<\delta<1 / 140 L$ by hypothesis, we can apply (5.1) to see that

$$
\left\|v_{j i}(x)-u_{j i}\right\|=\left\|\left(v_{i j}(x)\right)^{-1}-u_{i j}^{-1}\right\| \leq 2\left\|v_{i j}(x)-u_{i j}\right\|<140 L \delta .
$$

We conclude that $\beta(\pi)=\left\{v_{i j}\right\}$ is $C_{0}^{\prime \prime} \delta$-flat where $C_{0}^{\prime \prime}=280 L \delta$, completing the proof of Proposition 5.4 .

\section{Proofs of Theorems 3.1 and 3.3}

Most of the work needed to prove Theorems 3.1 and 3.3 was done in Sections 4 and 5 . What is left is basically bookkeeping related the various constants defined so far, but it is somewhat technical due to the nature of the definitions of $\alpha$ and $\beta$.

Proof of Theorem 3.1. The definitions of $\alpha$ and $\beta$ are given in Sections 4 and 5 . Propositions 4.6 and 5.4 show the existence of $\delta_{0}, \varepsilon_{0}>0$ and a constant $\max \left\{C_{0}^{\prime}, C_{0}^{\prime \prime}\right\}$ satisfying parts (1) and (2) of the theorem. We will actually set $C_{0}=\max \left\{C_{0}^{\prime}, 2 C_{0}^{\prime \prime}+40,4^{L+1}(K+1)\right\}$ where $K$ is provided by Lemma 4.7 .

We prove (3). Let $\pi=\alpha(\mathbf{v}), \pi^{\prime}=\alpha\left(\mathbf{v}^{\prime}\right)$. Let $\langle i, j\rangle \in \Lambda^{(1)}$ be such that

$$
\left\|\pi\left(\gamma_{i j}\right)-\pi^{\prime}\left(\gamma_{i j}\right)\right\|=\max _{\gamma \in \mathcal{F}_{\Lambda}}\left\|\pi(\gamma)-\pi^{\prime}(\gamma)\right\|=d\left(\pi, \pi^{\prime}\right)
$$

As in Definition 4.2, let $I=\left(i_{0}, \ldots, i\right)$ be the unique path along $T$ from $i_{0}$ to $i$ and $J=\left(i_{0}, \ldots, j\right)$ be the unique path from $i_{0}$ to $j$. Then

$$
\begin{aligned}
d\left(\pi, \pi^{\prime}\right) \leq & \left\|\tilde{\pi}\left(s\left(\gamma_{i j}\right)\right)-\tilde{\pi}(\langle i, j\rangle)\right\|+\left\|\tilde{\pi}^{\prime}(\langle i, j\rangle)-\tilde{\pi}^{\prime}\left(s\left(\gamma_{i j}\right)\right)\right\|+ \\
& +\left\|\tilde{\pi}(\langle i, j\rangle)-\tilde{\pi}^{\prime}(\langle i, j\rangle)\right\| \\
\leq & \left\|\tilde{\pi}\left(s\left(\gamma_{i j}\right)\langle i, j\rangle-1\right)-1\right\| \cdot\|\tilde{\pi}(\langle i, j\rangle)\|+ \\
& \quad+\left\|\tilde{\pi}^{\prime}\left(s\left(\gamma_{i j}\right)\langle i, j\rangle-1\right)-1\right\| \cdot\|\tilde{\pi}(\langle i, j\rangle)\|+\left\|\stackrel{\circ}{v}_{I} \stackrel{\circ}{v}_{i j} \stackrel{o}{v}_{J}^{-1}-\stackrel{\circ}{v}_{I}^{\prime} \stackrel{\circ}{i j}_{i j}^{\prime}\left(\dot{v}_{J}^{\prime}\right)^{-1}\right\| .
\end{aligned}
$$

From Lemma 4.7 we get that $\left\|\tilde{\pi}\left(s\left(\gamma_{i j}\right)\langle i, j\rangle^{-1}\right)-1\right\| \leq K \varepsilon$, where $K>0$ depends only on $\Lambda, T$, $i_{0}$, and $s$. The same bound holds with $\tilde{\pi}^{\prime}$ instead of $\tilde{\pi}$. Using this and the estimates $\left\|\dot{v}_{k l}\right\|<1+\varepsilon$, $\left\|\stackrel{\circ}{v}_{k l}-\stackrel{\circ}{v}_{k l}^{\prime}\right\|<d\left(\mathbf{v}, \mathbf{v}^{\prime}\right)$ for $\langle k, l\rangle \in \Lambda^{(1)}$, we see that

$$
d\left(\pi, \pi^{\prime}\right) \leq 2 \cdot K \varepsilon \cdot(1+\varepsilon)^{2 L+1}+(1+\varepsilon)^{2 L} d\left(\mathbf{v}, \mathbf{v}^{\prime}\right) .
$$

Since $(1+\varepsilon)^{2 L}<1+2^{2 L} \varepsilon$ and $d\left(\mathbf{v}, \mathbf{v}^{\prime}\right)<2+2 \varepsilon<4$ we have

$$
2 K \varepsilon(1+\varepsilon)^{2 L+1}+(1+\varepsilon)^{2 L} d\left(\mathbf{v}, \mathbf{v}^{\prime}\right)<2^{2 L+2} K \varepsilon+2^{2 L+2} \varepsilon+d\left(\mathbf{v}, \mathbf{v}^{\prime}\right) .
$$


Thus $d\left(\pi, \pi^{\prime}\right)<C_{0} \varepsilon+d\left(\mathbf{v}, \mathbf{v}^{\prime}\right)$.

For part (4), recall that $d\left(\pi, \pi^{\prime}\right)=\max _{\gamma \in \mathcal{F}_{\Lambda}}\|\pi(\gamma)-\pi(\gamma)\|$. Let $\mathbf{v}=\beta(\pi)$ and $\mathbf{v}^{\prime}=\beta\left(\pi^{\prime}\right)$ (these are $C_{0}^{\prime \prime} \delta$-flat GL $(A)$-coordinate bundles by Proposition 5.4). Recall that their definition (see Definition 5.3 makes use of the maps $\breve{\pi}$ and $\breve{\pi}^{\prime}$ (given by Proposition 5.6) respectively, and that $u_{i j}=\breve{\pi}\left(\gamma_{i j}\right)=\stackrel{\circ}{i j}_{i j}$ etc. For $\langle i, j\rangle \in \Lambda^{(1)}$ and $x \in c_{i j}$ we estimate

$$
\begin{aligned}
\left\|v_{i j}(x)-v_{i j}^{\prime}(x)\right\| \leq & \left\|v_{i j}(x)-u_{i j}\right\|+\left\|u_{i j}^{\prime}-v_{i j}^{\prime}(x)\right\|+\left\|u_{i j}-u_{i j}^{\prime}\right\| \\
< & C_{0}^{\prime \prime} \delta+C_{0}^{\prime \prime} \delta+\left\|u_{i j}-\pi\left(\gamma_{i j}\right)\right\|+\left\|\pi^{\prime}\left(\gamma_{i j}\right)-u_{i j}^{\prime}\right\|+ \\
& +\left\|\pi\left(\gamma_{i j}\right)-\pi^{\prime}\left(\gamma_{i j}\right)\right\| \\
< & 2 C_{0}^{\prime \prime} \delta+20 \delta+20 \delta+d\left(\pi, \pi^{\prime}\right) \\
& <C_{0} \delta+d\left(\pi, \pi^{\prime}\right) .
\end{aligned}
$$

It follows that $d\left(\mathbf{v}, \mathbf{v}^{\prime}\right)<C_{0} \delta+d\left(\pi, \pi^{\prime}\right)$.

Proof of Theorem 3.3. We prove (1) from the statement of the theorem first. Let $\varepsilon_{1}=1 / 140 L C_{0}$, $\delta_{1}=1 / 140 L C_{0}$, and $C_{1}=70 K C_{0}^{2}$. $\left(C_{0}\right.$ is provided by Theorem 3.1 , $K$ by Lemma 4.7 and $L$ by Notation 4.4). Let $0<\varepsilon<\varepsilon_{1}$ and suppose $\mathbf{v}=\left\{v_{i j}: c_{i j} \rightarrow \mathrm{GL}(A)\right\}$ is an $\varepsilon$-flat $\mathrm{GL}(A)$-coordinate bundle on $\Lambda$. Let $\pi=\alpha(\mathbf{v})$ and $\mathbf{v}^{\prime}=\left\{v_{i j}^{\prime}: c_{i j} \rightarrow \mathrm{GL}(A)\right\}=\beta(\pi)$. Observe that $\pi$ is an $\left(\mathcal{F}_{\Lambda}, C_{0} \varepsilon\right)$ representation and $C_{0} \varepsilon<1 / 140 L$ so that the construction of $\beta(\pi)$ from Section 5 may be used. We want to prove that

$$
d\left(\mathbf{v}, \mathbf{v}^{\prime}\right)=\max _{\langle i, j\rangle \in \Lambda^{(1)}} \max _{x \in c_{i j}}\left\|v_{i j}(x)-v_{i j}^{\prime}(x)\right\|<C_{1} \varepsilon .
$$

Recall the notation $\stackrel{\circ}{v}_{i j}=v_{i j}\left(\langle i, j\rangle^{\wedge}\right)$ from 4 .1. Since $\mathbf{v}$ is $\varepsilon$-flat and $\mathbf{v}^{\prime}$ is $C_{0}^{2} \varepsilon$-flat, it follows that

$$
d\left(\mathbf{v}, \mathbf{v}^{\prime}\right)<\max _{\langle i, j\rangle \in \Lambda^{(1)}}\left(\varepsilon+\left\|\stackrel{\circ}{v}_{i j}-\stackrel{\circ}{v}_{i j}^{\prime}\right\|+C_{0}^{2} \varepsilon\right) .
$$

Let $\langle i, j\rangle \in \Lambda^{(1)}$ and set $g_{i j}:=s\left(\gamma_{i j}\right) \cdot\langle i, j\rangle^{-1}$ as in Lemma 4.7. Applying the definition of $\tilde{\pi}$ (see Equation (4.1) and the fact that $\mathbf{v}$ is normalized (Definition 3.2), we obtain

$$
\pi\left(\gamma_{i j}\right)=\tilde{\pi}\left(s\left(\gamma_{i, j}\right)\right)=\tilde{\pi}(\langle i, j\rangle) \tilde{\pi}\left(g_{i j}\right)=\dot{\circ}_{i j} \tilde{\pi}\left(g_{i j}\right)
$$

The definition of $\beta(\pi)$ shows that $\stackrel{v}{i j}_{i}^{\prime}=\breve{\pi}\left(\gamma_{i j}\right)$ and Proposition 5.6 implies $\left\|\pi\left(\gamma_{i j}\right)-\breve{\pi}\left(\gamma_{i j}\right)\right\|<20 C_{0} \varepsilon$. Thus

$$
\begin{aligned}
\left\|\stackrel{v}{i j}_{i j}-\stackrel{\circ}{v}_{i j}^{\prime}\right\| & <\left\|\stackrel{\circ}{i j}_{i j}-\pi\left(\gamma_{i j}\right)\right\|+\left\|\pi\left(\gamma_{i j}\right)-\stackrel{\circ}{v}_{i j}^{\prime}\right\| \\
& =\left\|\stackrel{\circ}{i j}_{i j}\left(1-\tilde{\pi}\left(g_{i j}\right)\right)\right\|+\left\|\pi\left(\gamma_{i j}\right)-\tilde{\pi}\left(\gamma_{i j}\right)\right\| \\
& <\left\|\dot{v}_{i j}\right\|\left\|1-\tilde{\pi}\left(g_{i j}\right)\right\|+20 C_{0} \varepsilon \\
& <(1+\varepsilon)\left\|1-\tilde{\pi}\left(g_{i j}\right)\right\|+20 C_{0} \varepsilon .
\end{aligned}
$$

Lemma 4.7 guarantees that $\left\|1_{A}-\tilde{\pi}\left(g_{i j}\right)\right\|<K \varepsilon$. In combination with (6.1) and (6.2) this proves that

$$
d\left(\mathbf{v}, \mathbf{v}^{\prime}\right)<\varepsilon+C_{0}^{2} \varepsilon+(1+\varepsilon) K \varepsilon+20 C_{0} \varepsilon<C_{1} \varepsilon .
$$

We prove (2) from the statement of the theorem. Let $0<\delta<\delta_{1}$ and suppose $\pi: \Gamma \rightarrow \mathrm{GL}(A)$ is an $\left(\mathcal{F}_{\Lambda}, \delta\right)$-representation. Let $\mathbf{v}=\left\{v_{i j}\right\}=\beta(\pi)$ (this is a $C_{0} \delta$-flat $\mathrm{GL}(A)$-coordinate bundle) and let $\breve{\pi}: \Gamma \rightarrow \mathrm{U}(A)$ be given by Proposition 5.6. Let also $\pi^{\prime}=\alpha(\mathbf{v})$ (this is an $\left(\mathcal{F}_{\Lambda}, C_{0}^{2} \delta\right)$-representation of $\Gamma$ to $\operatorname{GL}(A))$. We want to prove that

$$
d\left(\pi, \pi^{\prime}\right)=\max _{\gamma \in \mathcal{F}_{\Lambda}}\left\|\pi(\gamma)-\pi^{\prime}(\gamma)\right\|<C_{1} \delta
$$


Suppose $\langle i, j\rangle \in \Lambda^{(1)}$. As above, we may write $s\left(\gamma_{i j}\right)=\langle i, j\rangle \cdot g_{i j}$ where by Lemma 4.7, $\| \tilde{\pi}^{\prime}\left(g_{i j}\right)-$ $1_{A} \|<K C_{0} \delta$.

First notice that $\stackrel{\circ}{i j}_{i j}=\breve{\pi}\left(\gamma_{i j}\right)$ by the definition of $\beta(\pi)=\mathbf{v}$ (Definition 5.3). Let $I$ be the unique path along $T$ from $i_{0}$ to $i$ and $J$ be the unique path from $i_{0}$ to $j$. Observe that $\mathbf{v}$ is normalized since $\breve{\pi}(e)=1_{A}$; hence $\stackrel{\circ}{v}_{I}=1_{A}=\stackrel{\circ}{v}_{J}$ because $I$ and $J$ are paths in the tree $T$. Then, by the definition of $\alpha(\mathbf{v})=\pi^{\prime}$

$$
\pi^{\prime}\left(\gamma_{i j}\right)=\tilde{\pi}^{\prime}\left(s\left(\gamma_{i, j}\right)\right)=\tilde{\pi}^{\prime}(\langle i, j\rangle) \cdot \tilde{\pi}^{\prime}(g)=\stackrel{\circ}{v}_{I} \stackrel{\circ}{i j}_{i j} \dot{o}_{J}^{-1} \cdot \tilde{\pi}^{\prime}(g)=\stackrel{\circ}{v}_{i j} \cdot \tilde{\pi}^{\prime}(g)=\breve{\pi}\left(\gamma_{i j}\right) \cdot \tilde{\pi}^{\prime}(g) .
$$

Therefore

$$
\begin{aligned}
\left\|\pi^{\prime}\left(\gamma_{i j}\right)-\pi\left(\gamma_{i j}\right)\right\| & \leq\left\|\pi^{\prime}\left(\gamma_{i j}\right)-\pi\left(\gamma_{i j}\right) \tilde{\pi}^{\prime}(g)\right\|+\left\|\pi\left(\gamma_{i j}\right) \tilde{\pi}^{\prime}(g)-\pi\left(\gamma_{i j}\right)\right\| \\
& \leq\left\|\breve{\pi}\left(\gamma_{i j}\right)-\pi\left(\gamma_{i j}\right)\right\|\left\|\tilde{\pi}^{\prime}(g)\right\|+\left\|\pi\left(\gamma_{i j}\right)\right\|\left\|\tilde{\pi}^{\prime}(g)-1_{A}\right\| \\
& <20 \delta\left(1+K C_{0}^{2} \delta\right)+(1+\delta)\left(K C_{0}^{2} \delta\right) \\
& <C_{1} \delta .
\end{aligned}
$$

\section{Almost flat bundles}

The goal of this section is to connect the notion of almost flat coordinate bundle from Definition 2.5. which is defined using simplicial structure and involves cocycles defined on closed sets, with the notion of almost flat bundle over a compact space from Definition 7.1 below.

Almost flat bundles and $K$-theory classes appeared in the work Gromov and Lawson [9], of Connes, Gromov, and Moscovici [3, 18, 21. In these references a vector bundle over a Riemannian manifold is called $\varepsilon$-flat if there is a metric-preserving connection with curvature of norm less than $\varepsilon$. Almost flat $K$-theory classes have been studied in different contexts in [2, 4, 5, 10, 16, 17]. We adapt the definition to bundles over topological spaces as in $[5]$ and connect this with the version for simplicial complexes by proving Proposition 7.3 .

Let $X$ be a compact space and let $\mathcal{V}=\left\{V_{i}\right\}$ be a finite open cover of $X$. A Čech 1-cocycle $\left\{v_{i j}: V_{i} \cap V_{j} \rightarrow \mathrm{GL}(A)\right\}$ satisfies $v_{i j}(x)=v_{j i}(x)^{-1}$ for all $x \in V_{i} \cap V_{j}$ and $v_{i k}(x)=v_{i j}(x) v_{j k}(x)$ for all $x \in V_{i} \cap V_{j} \cap V_{k}$.

7.1 Definition. Let $\varepsilon \geq 0$.

(1) A Čech 1-cocycle $\left\{v_{i j}: V_{i} \cap V_{j} \rightarrow \mathrm{GL}(A)\right\}$ is $\varepsilon$-flat, if

(a) $v_{i j}(x) \in \mathrm{U}(A)_{\varepsilon}$ for all $x \in V_{i} \cap V_{j}$; and

(b) $\left\|v_{i j}(x)-v_{i j}(y)\right\|<\varepsilon$ for all $x, y \in V_{i} \cap V_{j}$.

(2) A principal $\mathrm{GL}(A)$-bundle $E$ over $X$ is $(\mathcal{V}, \varepsilon)$-flat if its isomorphism class is represented by an $\varepsilon$-flat cocycle $\left\{v_{i j}: V_{i} \cap V_{j} \rightarrow \mathrm{GL}(A)\right\}$.

It is clear that if $\mathcal{V}^{\prime}$ is an open cover that refines $\mathcal{V}$, then the restriction of $\left\{v_{i j}\right\}$ to $\mathcal{V}^{\prime}$ is also $\varepsilon$-flat.

We now establish a result that connects the notion of $\varepsilon$-flat Čech 1-cocycles from Definition 7.1 with the notion of $\varepsilon$-flat coordinate bundle in the simplicial sense as given in Definition 2.5. Suppose that $X=|\Lambda|$ is the geometric realization of a finite simplicial complex $\Lambda$. Recall that $X$ has a (closed) cover $\mathcal{C}_{\Lambda}$ given by dual cells $c_{i}$; see Section 2. Let $d$ be the canonical metric for the topology of $X$ obtained using barycentric coordinates. Fix a sufficiently small number $\nu>0$ such that if we set $V_{i}=\left\{x \in X: \operatorname{dist}\left(x, c_{i}\right)<\nu\right\}$, then for any finite intersection

$$
V_{i_{1}} \cap V_{i_{2}} \cap \cdots \cap V_{i_{k}} \neq \emptyset \quad \Leftrightarrow \quad c_{i_{1}} \cap c_{i_{2}} \cap \cdots \cap c_{i_{k}} \neq \emptyset .
$$


Note that if $\left\{v_{i j}\right\}$ is as in Definition 7.1 and the cover $\left\{V_{i}\right\}$ satisfies (7.1), then the restriction of $\left\{v_{i j}\right\}$ to $c_{i} \cap c_{j} \subset V_{i} \cap V_{j}$ is an $\varepsilon$-flat coordinate bundle. Proposition 7.2 below allows us reverse this operation.

7.2 Proposition. There are numbers $\varepsilon_{0}>0$ and $r>0$, depending only on $\Lambda$, such that for any $0<\varepsilon<\varepsilon_{0}$, any $\varepsilon$-flat $\mathrm{GL}(A)$-coordinate bundle $\left\{v_{i j}: c_{i} \cap c_{j} \rightarrow U(A)_{\varepsilon}\right\}$ on $\Lambda$, and any $\nu>0$ satisfying (7.1), there is an re-flat cocycle $\left\{\widetilde{v}_{i j}: V_{i} \cap V_{j} \rightarrow U(A)_{r \varepsilon}\right\}$ that extends $v_{i j}$.

Proposition 7.2 is a direct consequence of Proposition 7.3 below, whose content is of independent interest in connection with extension properties of principal bundles.

Let $Y$ be a closed subspace of a compact metric space $X$ and let $\left\{U_{i}\right\}_{i=1}^{n}$ be a closed cover of $Y$. For $\nu>0$ and $i \in\{1, \ldots, n\}$ let

$$
U_{i}^{\nu}:=\left\{x \in X: \operatorname{dist}\left(x, U_{i}\right) \leq \nu\right\}
$$

and set $\tilde{Y}=\bigcup_{i=1}^{n} U_{i}^{\nu}$. Fix $\nu>0$ small enough such that for any finite intersection

$$
U_{i_{1}}^{\nu} \cap U_{i_{2}}^{\nu} \cap \cdots \cap U_{i_{k}}^{\nu} \neq \emptyset \quad \Leftrightarrow \quad U_{i_{1}} \cap U_{i_{2}} \cap \cdots \cap U_{i_{k}} \neq \emptyset
$$

\subsection{Proposition.}

(1) For any cocycle $v_{i j}: U_{i} \cap U_{j} \rightarrow G L(A)$ on $Y$ there exist $\nu>0$ satisfying (7.2) and a cocycle $\widetilde{v}_{i j}: U_{i}^{\nu} \cap U_{j}^{\nu} \rightarrow \mathrm{GL}(A)$ that extends $v_{i j}$, i.e. $\widetilde{v}_{i j}=v_{i j}$ on $U_{i} \cap U_{j}$.

(2) There exist $\varepsilon_{0} \in(0,1)$ and a universal constant $r=r_{n}$ that depends only on $n$ such that for any $0<\varepsilon<\varepsilon_{0}$, any $\varepsilon$-flat cocycle $v_{i j}: U_{i} \cap U_{j} \rightarrow U(A)_{\varepsilon}$ on $Y$, and any $\nu>0$ satisfying 7.2 , there is an $r \varepsilon$-flat cocycle $\widetilde{v}_{i j}: U_{i}^{\nu} \cap U_{j}^{\nu} \rightarrow U(A)_{r \varepsilon}$ on $\widetilde{Y}$ which extends $v_{i j}$.

Proof. We begin with the proof of (1) and will explain subsequently how to adapt the argument to prove (2) as well. We prove (1) by induction on the cardinality $n$ of the cover. Suppose that the statement is true for any integer $\leq n-1$. Let $v_{i j}: U_{i} \cap U_{j} \rightarrow \operatorname{GL}(A)$ be given with $1 \leq i, j \leq n$. Set $Y_{n-1}:=\bigcup_{i=1}^{n-1} U_{i}$. By the inductive hypothesis, there exist $\nu>0$ satisfying (7.2) and a cocycle $\widetilde{v}_{i j}: U_{i}^{\nu} \cap U_{j}^{\nu} \rightarrow \mathrm{GL}(A), 1 \leq i, j \leq n-1$, which extends $v_{i j}$. Thus the following condition, labelled as $(n-1)$, is satisfied:

$$
\widetilde{v}_{r s}=\widetilde{v}_{r t} \widetilde{v}_{t s} \text { on } U_{r}^{\nu} \cap U_{t}^{\nu} \cap U_{s}^{\nu} \text { for all } 1 \leq r \leq t \leq s \leq n-1
$$

To pass from $n-1$ to $n$ we proceed again by induction on increasing $k \in L_{n}$, where $L_{n}$ is the set of those integers $1 \leq k \leq n$ with the property that $U_{k} \cap U_{n} \neq \emptyset$. The inductive hypothesis that we make is that the functions $\left\{v_{i n}: i \leq k, i \in L_{n}\right\}$ extend to functions $\widetilde{v}_{i n}: U_{i}^{\nu} \cap U_{n}^{\nu} \rightarrow G L(A)$ such that the following conditions (depending on $k$ ) are satisfied:

$$
\begin{gathered}
\widetilde{v}_{i n}=\widetilde{v}_{i j} \widetilde{v}_{j n} \text { on } U_{i}^{\nu} \cap U_{j}^{\nu} \cap U_{n}^{\nu} \text { for } i, j \leq k \text { with } i, j \in L_{n} . \\
\widetilde{v}_{i n}=\widetilde{v}_{i j} v_{j n} \text { on } U_{i}^{\nu} \cap U_{j} \cap U_{n} \text { for } i \leq k \leq j \text { with } i, j \in L_{n} .
\end{gathered}
$$

If $L_{n}$ reduces to $\{n\}$, then we simply define $\widetilde{v}_{n n}=1$ and we are done. Assume $L_{n}$ contains more than one element.

Let $\ell$ be the smallest element of $L_{n}($ so $\ell<n)$. To construct $\widetilde{v}_{\ell n}$ we first define an extension $v_{\ell n}^{\prime}$ of $v_{\ell n}$ on suitable closed subsets of $U_{\ell}^{\nu} \cap U_{n}^{\nu}$ as follows:

$$
v_{\ell n}^{\prime}=\widetilde{v}_{\ell j} v_{j n} \text { on } U_{\ell}^{\nu} \cap U_{j} \cap U_{n} \text { for all } \ell \leq j<n, j \in L_{n} \text {. }
$$


Let us observe that $v_{\ell n}^{\prime}$ is well-defined since if $\ell \leq i \leq j<n, i, j \in L_{n}$, then $\widetilde{v}_{\ell i} v_{i n}=\widetilde{v}_{\ell j} v_{j n}$ on $U_{\ell}^{\nu} \cap U_{i} \cap U_{j} \cap U_{n}$ if and only if $v_{i n}=\widetilde{v}_{i, \ell} \widetilde{v}_{\ell j} v_{j n}$ on the same set. In view of condition $(n-1)$ this reduces to the equality $v_{i n}=\widetilde{v}_{i j} v_{j n}$ on $U_{i} \cap U_{j} \cap U_{n}$, which holds true since $\widetilde{v}_{i j}$ extends $v_{i j}$. By Tietze's theorem we can now extend the function $v_{\ell n}^{\prime}$ defined by $\left(0^{\prime}\right)$ to a continuous function $\widetilde{v}_{\ell n}: U_{\ell}^{\nu} \cap U_{n}^{\nu} \rightarrow A$. Since GL(A) is open in $A$ we will have that $\widetilde{v}_{\ell n}(x) \in \operatorname{GL}(A)$ for all $x \in U_{\ell}^{\nu} \cap U_{n}^{\nu}$ provided that $\nu$ is sufficiently small. We need to very that $\widetilde{v}_{\ell n}$ satisfies $(1, \ell)$ and $(2, \ell)$. Condition $(1, \ell)$ amounts to $\widetilde{v}_{\ell n}=\widetilde{v}_{\ell \ell} \widetilde{v}_{\ell n}$ on $U_{\ell}^{\nu} \cap U_{n}^{\nu}$ which holds since $\widetilde{v}_{\ell \ell}=1$. Condition $(2, \ell)$ reduces to $\widetilde{v}_{\ell n}=\widetilde{v}_{\ell j} v_{j n}$ on $U_{\ell}^{\nu} \cap U_{j} \cap U_{n}$ for $\ell \leq j$ with $j \in L_{n}$. This holds true in view of $\left(0^{\prime}\right)$ and so the base case for the induction is complete.

Fix $k \in L_{n}, k<n$ and suppose now that we have constructed $\widetilde{v}_{i n}: U_{i}^{\nu} \cap U_{n}^{\nu} \rightarrow \operatorname{GL}(A)$ for all $i \in L_{n}$ with $i \leq k$ such that the conditions $(1, k)$ and $(2, k)$ are satisfied. Let $\ell \in L_{n}$ be the successor of $k$ in $L_{n}$. We may assume that $\ell<n$ for otherwise there is nothing to prove. We construct a map $\widetilde{v}_{\ell n}$ on $U_{\ell}^{\nu} \cap U_{n}^{\nu}$ that satisfies the corresponding conditions $(1, \ell)$ and $(2, \ell)$ as follows. The first step is to define an extension $v_{\ell n}^{\prime}$ of $v_{\ell n}$ on suitable closed subsets of $U_{\ell}^{\nu} \cap U_{n}^{\nu}$ as follows:

$$
\begin{gathered}
v_{\ell n}^{\prime}=\widetilde{v}_{\ell i} \widetilde{v}_{i n} \text { on } U_{\ell}^{\nu} \cap U_{i}^{\nu} \cap U_{n}^{\nu} \text { for all } i \leq k, i \in L_{n} . \\
v_{\ell n}^{\prime}=\widetilde{v}_{\ell j} v_{j n} \text { on } U_{\ell}^{\nu} \cap U_{j} \cap U_{n} \text { for all } \ell \leq j<n, j \in L_{n} .
\end{gathered}
$$

We need to observe that the conditions $\left(1^{\prime}\right)$ and $\left(2^{\prime}\right)$ are compatible so that $v_{\ell, n}^{\prime}$ is well-defined and continuous. There are three cases to verify. First we check that $\widetilde{v}_{\ell, i} \widetilde{v}_{i n}=\widetilde{v}_{\ell j} \widetilde{v}_{j n}$ on $U_{\ell}^{\nu} \cap U_{i}^{\nu} \cap U_{j}^{\nu} \cap U_{n}^{\nu}$ for $i, j \leq k, i, j \in L_{n}$. This is a consequence of conditions $(n-1)$ and $(1, k)$. Second, we verify that $\widetilde{v}_{\ell i} v_{i n}=\widetilde{v}_{\ell j} v_{j n}$ on $U_{\ell}^{\nu} \cap U_{i} \cap U_{j} \cap U_{n}$ for $\ell \leq i, j<n, i, j \in L_{n}$. Note that this holds if and only if $v_{i n}=\widetilde{v}_{i, \ell} \widetilde{v}_{\ell j} v_{j n}$ on the same set. In view of condition $(n-1)$ this reduces to the equality $v_{i n}=\widetilde{v}_{i j} v_{j n}$ on $U_{i} \cap U_{j} \cap U_{n}$, which holds true since $\widetilde{v}_{i j}$ extends $v_{i j}$. Finally we need to verify that $\widetilde{v}_{\ell i} \widetilde{v}_{i n}=\widetilde{v}_{\ell j} v_{j n}$ on $\left(U_{\ell}^{\nu} \cap U_{i}^{\nu} \cap U_{n}^{\nu}\right) \cap\left(U_{\ell}^{\nu} \cap U_{j} \cap U_{n}\right)=U_{\ell}^{\nu} \cap U_{i}^{\nu} \cap U_{j} \cap U_{n}$ for $i \leq k<\ell \leq j<n, i, j \in L_{n}$. By $(n-1)$, this equality holds if and only if $\widetilde{v}_{i n}=\widetilde{v}_{i j} v_{j n}$ on $U_{i}^{\nu} \cap U_{j} \cap U_{n}$. The latter equality holds due to condition $(2, k)$ which is satisfied by the inductive hypothesis. By Tietze's theorem we can now extend the function $v_{\ell n}^{\prime}$ defined by $\left(1^{\prime}\right)$ and $\left(2^{\prime}\right)$ to a continuous function $\widetilde{v}_{\ell n}: U_{\ell}^{\nu} \cap U_{n}^{\nu} \rightarrow A$. Since $\mathrm{GL}(A)$ is open in $A$ we will have that $\widetilde{v}_{\ell n}(x) \in \mathrm{GL}(A)$ for all $U_{\ell}^{\nu} \cap U_{n}^{\nu}$ provided that $\nu$ is sufficiently small. It is clear that the functions $\left(\widetilde{v}_{i n}\right)_{i \leq \ell}$ satisfy the conditions $(1, \ell),(2, \ell)$ as a consequence of $\left(1^{\prime}\right),(1, k),\left(2^{\prime}\right)$ and $(2, k)$. This completes the inductive step from $k$ to $\ell$ and hence from $n-1$ to $n$. During this step we had to pass to a possibly smaller $\nu$ but this does not affect the conclusion.

(2). The proof follows the pattern of the proof of (1) with one important modification. Namely we use the following strengthened version of Tietze's theorem due to Dugunji [6]. Let $X$ be an arbitrary metric space, $Y$ a closed subset of $X, A$ a locally convex linear space and $f: Y \rightarrow A$ a continuous map. Then there exists an extension $\tilde{f}: X \rightarrow A$ of $f$ such that $\tilde{f}(X)$ is contained in the convex hull of $f(Y)$.

Fix a point $x_{i j}$ in each nonempty intersection $U_{i} \cap U_{j}$ and set $\stackrel{\circ}{i j}_{i j}:=v_{i j}\left(x_{i j}\right)$. Since the cocycle is $\varepsilon$-flat, we have that $\left\|v_{i j}(x)-\stackrel{\circ}{i j}_{i j}\right\|<\varepsilon$.

Let us define positive numbers $r(i, j)$ for $1 \leq i \leq j \leq n$ as follows. If $i=j$, then $r(i, j)=1$. If $i<j, r(i, j)$ is defined by the following recurrence formula. Set $r_{k}=\max \{r(i, j): 1 \leq i \leq j \leq k\}$ and $r_{1}=1$. If $1 \leq \ell<n$ then we define $r(\ell, n)=\left(3 r_{n-1}+7\right) \max \{r(i, n): 1 \leq i<\ell\}$ with the convention that $\max \emptyset=1$.

We only need to consider the maps $\widetilde{v}_{\ell n}$ with $\ell \in L_{n}=\left\{i: U_{i} \cap U_{n} \neq \emptyset\right\}$ and $\ell<n$. We proceed as in proof of (1) by induction on $n$ and $k \in L_{n}$ with the additional provision that

$\left(3^{\prime}\right) \widetilde{v}_{i j}\left(U_{i}^{\nu} \cap U_{j}^{\nu}\right) \subset B\left(\stackrel{\circ}{i j}_{i j}, r(i, j) \varepsilon\right)$, for all $1 \leq i \leq j \leq n-1$ and for all $(i, j)$ with $i<k, i \in L_{n}$ and $j=n$. 
The basic idea of the proof is to observe that it follows from the equations $\left(0^{\prime}\right),\left(1^{\prime}\right)$ and $\left(2^{\prime}\right)$ that $v_{\ell n}^{\prime}$ is close to $\widetilde{v}_{\ell i} \widetilde{v}_{i n}$ (if $i<\ell$ ) or $\widetilde{v}_{\ell i} v_{i n}$ (if $\ell \leq i$ ) both of which are near $\dot{v}_{\ell i} \dot{v}_{i n}$ and hence $v_{\ell, n}^{\prime}$ is close to $\dot{v}_{\ell n}$. It will follow that the image of $v_{\ell n}^{\prime}$ is contained in a ball $B\left(\dot{v}_{\ell n}, r(\ell, n) \varepsilon\right)$ where $r(\ell, n)$ is a universal constant computed recursively from previously determined $r(i, j)$. Therefore we can invoke the strengthened version of Tietze's theorem of $\left[6\right.$ to extend $v_{\ell, n}^{\prime}$ to a continuous map $\widetilde{v}_{\ell, n}$ with values in convex open ball $B\left(\dot{v}_{\ell n}, r(\ell, n) \varepsilon\right)$.

Fix $k \in L_{n}, k<n$. By the inductive hypothesis, suppose that we have constructed $\widetilde{v}_{i j}$ and they satisfy $\left(3^{\prime}\right)$. We need to consider two cases. The first is the case when $k=\min L_{n}$. Letting $\ell=k$ and $0<\varepsilon<1$, then from condition $\left(0^{\prime}\right)$, for each $x \in U_{\ell}^{\nu} \cap U_{j} \cap U_{n}$ with $\ell \leq j<n, j \in L_{n}$ :

$$
\begin{aligned}
\left\|v_{\ell n}^{\prime}(x)-\dot{v}_{\ell n}\right\| \leq & \left\|\widetilde{v}_{\ell j}(x)-\dot{v}_{\ell j}\right\|\left\|v_{j n}(x)\right\|+\left\|\dot{\circ}_{\ell j}\right\|\left\|v_{j n}(x)-\dot{v}_{i n}\right\|+ \\
& +\left\|\dot{v}_{\ell j}-\stackrel{\circ}{j n}_{\dot{v}_{\ell n}}\right\| \\
< & r(\ell, i) \varepsilon(1+\varepsilon)+\varepsilon(1+\varepsilon)+\varepsilon(3+2 \varepsilon) \\
\leq & \left(3 r_{n-1}+7\right) \varepsilon \leq r(\ell, n) \varepsilon .
\end{aligned}
$$

Let $\ell$ be the successor of $k$ in $L_{n}$. We may assume that $\ell<n$ otherwise we are done. If $x \in U_{\ell}^{\nu} \cap U_{j} \cap U_{n}$ with $\ell \leq j<n, j \in L_{n}$, then using $\left(2^{\prime}\right)$ it follows just as above that $\left\|v_{\ell n}^{\prime}(x)-\dot{v}_{\ell n}\right\| \leq$ $\left(3 r_{n-1}+7\right) \varepsilon=r(\ell, n) \varepsilon$. On the other hand, if $x \in U_{\ell}^{\nu} \cap U_{i}^{\nu} \cap U_{n}$ with $i<\ell, i \in L_{n}$, then using $\left(1^{\prime}\right)$ we have

$$
\begin{aligned}
\left\|v_{\ell n}^{\prime}(x)-\dot{\circ}_{\ell n}\right\| & \leq\left\|\widetilde{v}_{\ell i}(x)-\stackrel{\circ}{\ell i}_{\ell}\right\|\left\|\widetilde{v}_{i n}(x)\right\|+\left\|\stackrel{\circ}{\ell i}_{\ell}\right\|\left\|\widetilde{v}_{i n}(x)-\stackrel{\circ}{i n}_{i n}\right\|+\left\|\dot{\circ}_{\ell i}-\stackrel{\circ}{i n}_{i n} \stackrel{v}{\ell n}\right\| \\
& <r(\ell, i) \varepsilon(1+(r(i, n)+1) \varepsilon)+(1+\varepsilon) r(i, n) \varepsilon+\varepsilon(2+3 \varepsilon) \\
& \leq(3 r(\ell, i)+7) r(i, n) \\
& \leq\left(3 r_{n-1}+7\right) \max \left\{r(i, n): i<\ell, i \in L_{n}\right\} \\
& \leq r(\ell, n) \varepsilon .
\end{aligned}
$$

In view of this estimates we can extend $v_{\ell n}^{\prime}$ to $\widetilde{v}_{\ell n}$ using the strengthened version of Tietze's theorem so that $\widetilde{v}_{\ell n}\left(U_{\ell}^{\nu} \cap U_{n}^{\nu}\right) \subset B\left({\stackrel{\circ}{v_{\ell}}}_{1}, r(\ell, n) \varepsilon\right)$. It follows that $\left\|\widetilde{v}_{i j}(x)-\stackrel{\circ}{i j}_{i j}\right\|<r_{n} \varepsilon$ for all $x \in U_{i}^{\nu} \cap U_{j}^{\nu}$. This completes the proof.

\section{Almost flat K-theory classes and the K-theoretical MF-property}

One of the motivations for this paper is the detection of nontrivial K-theory elements of a group $\mathrm{C}^{*}$ algebra, via lifting of homomorphisms $K_{0}\left(C^{*}(\Gamma)\right) \rightarrow \mathbb{Z}$ to quasi-representations $C^{*}(\Gamma) \rightarrow M_{m}(\mathbb{C})$. Suppose that the full assembly map is a bijection for a discrete group $\Gamma$. Roughly speaking, our main result states that the quasi-representations $C^{*}(\Gamma) \rightarrow M_{m}(\mathbb{C})$ which induce interesting partial maps on K-theory are as abundant as the non-trivial almost flat K-theory classes of the classifying space $B \Gamma$. More generally, for a $\mathrm{C}^{*}$-algebra $B$ we consider the connection between almost flat $\mathrm{K}$ theory classes in $K_{0}(C(B \Gamma) \otimes B)$ and quasi-representations $C^{*}(\Gamma) \rightarrow M_{m}(B)$ that implement a given homomorphism $K_{*}\left(C^{*}(\Gamma)\right) \rightarrow K_{*}(B)$.

Let $A$ be a unital $\mathrm{C}^{*}$-algebra. A quasi-representation $\pi: \Gamma \rightarrow \mathrm{GL}(A)$ extends to a unital linear contraction $\pi: \ell^{1}(\Gamma) \rightarrow A$ in the obvious way. We like to think of $\pi$ as "inducing" a partially defined map $\pi_{\sharp}: K_{0}\left(\ell^{1}(\Gamma)\right) \rightarrow K_{0}(A)$ (cf. 4,5$\left.]\right)$. We briefly recall the definition of $\pi_{\sharp}$. In the definition we write $\chi$ for the function $\zeta \mapsto \frac{1}{2 \pi i} \int_{C}(z-\zeta)^{-1} d z$, where $C=\{z \in \mathbb{C}:|z-1|=1 / 4\}$.

8.1 Definition (c.f. [4]). Let $D, B$ be Banach algebras and let $\pi: D \rightarrow B$ be a unital contractive map. Let $p \in M_{m}(\mathbb{C}) \otimes D$ be an idempotent and let $x=\left(\operatorname{id}_{m} \otimes \pi\right)(p) \in M_{m}(\mathbb{C}) \otimes B$. Define 
$\pi_{\sharp}(p)=[\chi(x)] \in K_{0}(B)$ whenever $\left\|x^{2}-x\right\|<1 / 4$. In a similar manner, one defines the pushforward $\pi_{\sharp}(u) \in K_{1}(B)$ of an invertible element $u \in M_{m}(\mathbb{C}) \otimes D$ as the class of $\left[\left(\operatorname{id}_{m} \otimes \pi\right)(u)\right]$, under the assumption that $\pi$ is (sufficiently) approximately multiplicative on a suitable finite subset of $D$ that depends on $u$.

In general $\pi_{\sharp}(p)$ is not necessarily equal to $\pi_{\sharp}(q)$ if $[p]=[q]$ in $K_{0}(D)$. To bypass this nuisance, we use a discrete version of the asymptotic homomorphisms of Connes and Higson.

A discrete asymptotic homomorphism from an involutive Banach algebra $D$ to $\mathrm{C}^{*}$-algebras $B_{n}$ consists of a sequence $\left\{\pi_{n}: D \rightarrow B_{n}\right\}_{n=1}^{\infty}$ of maps such that

$$
\lim _{n \rightarrow \infty}\left\{\begin{array}{l}
\left\|\pi_{n}\left(a+\lambda a^{\prime}\right)-\pi_{n}(a)-\lambda \pi_{n}\left(a^{\prime}\right)\right\| \\
\left\|\pi_{n}\left(a^{*}\right)-\pi_{n}(a)^{*}\right\| \\
\left\|\pi_{n}\left(a a^{\prime}\right)-\pi_{n}(a) \pi_{n}\left(a^{\prime}\right)\right\|
\end{array}\right\}=0
$$

for all $a, a^{\prime} \in D$ and $\lambda \in \mathbb{C}$. The sequence $\left\{\pi_{n}\right\}_{n}$ induces a $*$-homomorphism $D \rightarrow \prod_{n=1}^{\infty} B_{n} / \sum_{n=1}^{\infty} B_{n}$. If each $B_{n}$ is a matrix algebra over some fixed $\mathrm{C}^{*}$-algebra $B$, then this further induces a group homomorphism

$$
K_{*}(D) \rightarrow \prod_{n=1}^{\infty} K_{*}(B) / \sum_{n=1}^{\infty} K_{*}(B) .
$$

A discrete asymptotic homomorphism gives a canonical way to push forward an element $x \in K_{*}(D)$ to a sequence $\left(\pi_{n \sharp}(x)\right)$ of elements of $K_{*}(B)$, which is well-defined up to tail equivalence: two sequences are tail equivalent, written $\left(y_{n}\right) \equiv\left(z_{n}\right)$, if there is $m$ such that $y_{n}=z_{n}$ for all $n \geq m$. Note that one can adapt Definition 8.1 to maps which are approximately contractive (in addition to being approximately multiplicative).

8.2 Remark. Let $\Gamma$ be a discrete countable group with a finite set of generators $\mathcal{F}$. We need the following observations:

(1) A sequence of $\left(\mathcal{F}, \delta_{n}\right)$-representations $\left\{\pi_{n}: \Gamma \rightarrow U\left(B_{n}\right)\right\}_{n=1}^{\infty}$, with $\delta_{n} \rightarrow 0$ as $n \rightarrow \infty$, induces a discrete asymptotic homomorphism (still written $\left(\pi_{n}\right)_{n=1}^{\infty}$ ) from the involutive Banach algebra $\ell^{1}(\Gamma)$ to the $\mathrm{C}^{*}$-algebras $B_{n}$.

(2) A discrete asymptotic homomorphism $\left\{\pi_{n}: \ell^{1}(\Gamma) \rightarrow B_{n}\right\}_{n=1}^{\infty}$ as above induces a $*$-homomorphism $\pi_{\infty}: \ell^{1}(\Gamma) \rightarrow B_{\infty}:=\prod_{n=1}^{\infty} B_{n} / \sum_{n=1}^{\infty} B_{n}$ and hence a $*$-homomorphism $\bar{\pi}_{\infty}: C^{*}(\Gamma) \rightarrow B_{\infty}$ such as the following diagram is commutative.

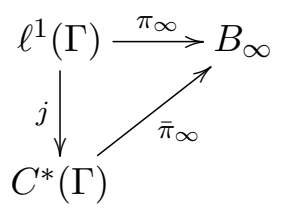

(where $j$ is the canonical map).

(3) Let $\left\{\bar{\pi}_{n}: C^{*}(\Gamma) \rightarrow B_{n}\right\}_{n=1}^{\infty}$ be a discrete asymptotic homomorphism given by some settheoretic lift of $\bar{\pi}_{\infty}$. If $y \in K_{*}\left(\ell^{1}(\Gamma)\right)$, then we have the following tail equivalence:

$$
\left(\bar{\pi}_{n \sharp}\left(j_{*}(y)\right)\right)_{n=1}^{\infty} \equiv\left(\pi_{n \sharp}(y)\right)_{n=1}^{\infty}
$$


The results of [4] will allow us to relate the push-forward of elements in the image of the full Baum-Connes assembly map with the almost flat bundles we have constructed. The relationshp involves the Mishchenko line bundle and its push-forward, which we now discuss.

Let $\widetilde{X}$ be the universal cover of $X=|\Lambda|$. Consider the dual cover $\mathcal{C}_{\Lambda}=\left\{c_{i}\right\}_{i}$ of $X$ and the associated open cover $\mathcal{V}_{\nu}=\left\{V_{i}\right\}_{i}$ where $V_{i}=\left\{x \in X: d\left(x, c_{i}\right)<\nu\right\}$. The Mishchenko line bundle is the bundle $\widetilde{X} \times_{\Gamma} \ell^{1}(\Gamma) \rightarrow X$, obtained from $\widetilde{X} \times \ell^{1}(\Gamma)$ by passing to the quotient with respect to the diagonal action of $\Gamma$. It is isomorphic to the bundle $E$ obtained from the disjoint union $\bigsqcup V_{i} \times \ell^{1}(\Gamma)$ by identifying $(x, a)$ with $\left(x, \gamma_{i j} a\right)$ whenever $x \in V_{i} \cap V_{j}$, where $\gamma_{i j} \in \Gamma$ are as in Notation 2.1 see for example [2, Lemma 3.3]. Let $\left\{\chi_{i}\right\}$ be a partition of unity subordinate to $\left\{V_{i}\right\}$. It follows that the Mishchenko line bundle corresponds to the class of the projection

$$
e:=\sum_{i, j} e_{i j} \otimes \chi_{i}^{1 / 2} \chi_{j}^{1 / 2} \otimes \gamma_{i j} \in M_{N}(\mathbb{C}) \otimes C(X) \otimes \mathbb{C}[\Gamma]
$$

where $\left\{e_{i j}\right\}$ are the canonical matrix units of $M_{N}(\mathbb{C})$ and $N$ is the number of vertices in $\Lambda$. We have inclusions of rings $\mathbb{C}[\Gamma] \subset \ell^{1}(\Gamma) \subset C^{*}(\Gamma)$. The class of the idempotent $e$ in $K_{0}\left(C(X) \otimes \ell^{1}(\Gamma)\right)$ or $K_{0}\left(C(X) \otimes C^{*}(\Gamma)\right)$ is denoted by $\ell$.

8.3 Notation. For an $\left(\mathcal{F}_{\Lambda}, \varepsilon\right)$-representation $\pi: \Gamma \rightarrow U(A)$ as in Definition 2.2, we set

$$
\ell_{\pi}:=\left(\operatorname{id}_{C(X)} \otimes \pi\right)_{\sharp}(e) \in K_{0}(C(X) \otimes A) .
$$

From Definition 5.3 we get the coordinate bundle $\beta(\pi)$ associated with $\pi$. Applying Proposition 7.3 to $\beta(\pi)$ we obtain an almost flat cocycle $\left\{v_{i j}: V_{i} \cap V_{j} \rightarrow \operatorname{GL}(A)\right\}$. Let $E_{\pi}$ be the bundle constructed from the disjoint union $\bigsqcup V_{i} \times A$ by identifying $(x, a)$ with $\left(x, v_{i j}(x) a\right)$ for $x$ in $V_{i j}$.

8.4 Proposition. There is $\varepsilon_{0}$ such that, for any $0<\varepsilon<\varepsilon_{0}$ and any $\left(\mathcal{F}_{\Lambda}, \varepsilon\right)$-representation $\pi: \Gamma \rightarrow U(A)$,

$$
\left[E_{\pi}\right]=\ell_{\pi} \in K_{0}(C(X) \otimes A) .
$$

Proof. Let $\varepsilon>0$ and let $\pi: \Gamma \rightarrow U(A)$ be an $\left(\mathcal{F}_{\Lambda}, \varepsilon\right)$-representation. Because the bundle $E_{\pi}$ is represented by the idempotent $p=\sum_{i, j} e_{i j} \otimes \chi_{i}^{1 / 2} \chi_{j}^{1 / 2} \otimes v_{i j}$, it follows that

$$
p-\left(\operatorname{id}_{C(X)} \otimes \pi\right)(e)=\sum_{i, j} e_{i j} \otimes \chi_{i}^{1 / 2} \chi_{j}^{1 / 2} \otimes\left(\pi\left(\gamma_{i j}\right)-v_{i j}\right)
$$

Now, by the construction of $\left\{v_{i j}\right\}$, there is a constant $C$ depending only on $\Lambda$ such that $\sup _{x \in V_{i} \cap V_{j}} \| \pi\left(\gamma_{i j}\right)-$ $v_{i j}(x) \|<C \varepsilon$. Therefore, $\left\|p-\left(\operatorname{id}_{C(X)} \otimes \pi\right)(e)\right\|<1 / 4$ if $\varepsilon_{0}$ is chosen to be sufficiently small.

Let $X$ be a compact connected space and let $B$ be a unital $\mathrm{C}^{*}$-algebra. We consider locally trivial bundles $E$ over $X$ with fiber finitely generated projective Hilbert-modules $F$ over $B$ and structure group $\mathrm{GL}(A)$, where $A=L_{B}(F)$, the $\mathrm{C}^{*}$-algebra of $B$-linear adjointable endomorphisms of $F$. The K-theory group $K_{0}(C(X) \otimes B)$ consists of formal differences of isomorphism classes of such bundles. Let $\mathcal{V}$ be a finite open cover of $X$. A bundle $E$ as above is $(\mathcal{V}, \varepsilon)$-flat if it admits an $(\mathcal{V}, \varepsilon)$-flat associated $\mathrm{GL}(A)$-principal bundle in the sense of Definition $7.1(2)$.

8.5 Definition. An element $x \in K_{0}(C(X) \otimes B)$ is almost flat if there is a finite open cover $\mathcal{V}$ of $X$ such that for every $\varepsilon>0$ there are $(\mathcal{V}, \varepsilon)$-flat bundles $E^{ \pm}$over $X$ such that $\alpha=\left[E^{+}\right]-\left[E^{-}\right]$. We say that $x \in K_{0}(C(X) \otimes B)$ is almost flat modulo torsion if there is a torsion element $t \in K_{0}(C(X) \otimes B)$ such that $x-t$ is almost flat. 
By the UCT given in [14, Lemma 3.4], the Kasparov product

$$
K K(\mathbb{C}, C(B \Gamma) \otimes B) \times K K_{*}(C(B \Gamma), \mathbb{C}) \rightarrow K K_{*}(\mathbb{C}, B), \quad(x, z) \mapsto\langle x, z\rangle,
$$

induces an exact sequence

$$
\operatorname{Ext}\left(K_{*}(B \Gamma), K_{*+1}(B)\right) \longmapsto K_{0}(C(B \Gamma) \otimes B) \rightarrow \operatorname{Hom}\left(K_{*}(B \Gamma), K_{*}(B)\right) .
$$

If $K_{*}(B)$ is finitely generated and torsion free, then the torsion subgroup of $K_{0}(C(B \Gamma) \otimes B)$ coincides with the image of $\operatorname{Ext}\left(K_{*}(B \Gamma), K_{*+1}(B)\right)$.

8.6 Theorem. Let $\Gamma$ be a discrete countable group whose classifying space $B \Gamma$ is a finite simplicial complex and let $B$ be a unital $C^{*}$-algebra. Consider the following conditions:

(1) For any $x \in K_{0}(C(B \Gamma) \otimes B)$ there is $t \in \operatorname{Ext}\left(K_{*}(B \Gamma), K_{*+1}(B)\right)$ such that $x-t$ is almost flat.

(2) For any group homomorphism $h: K_{*}\left(C^{*}(\Gamma)\right) \rightarrow K_{*}(B)$ there exist discrete asymptotic homomorphisms $\left\{\pi_{n}^{ \pm}: C^{*}(\Gamma) \rightarrow M_{k(n)}(B)\right\}_{n}$ such that $\left(\pi_{n \sharp}^{+}(y)-\pi_{n \sharp}^{-}(y)\right) \equiv(h(y))$ for every $y$ in the image of the full assembly map $\mu: K_{*}(B \Gamma) \rightarrow K_{*}\left(C^{*}(\Gamma)\right)$.

Then (1) $\Rightarrow$ (2). Moreover if $K_{*}(B)$ is finitely generated and if $\mu$ is split injective, then (2) $\Rightarrow$ (1).

Proof. $(1) \Rightarrow(2)$. Let $h: K_{*}\left(C^{*}(\Gamma)\right) \rightarrow K_{*}(B)$ be given. Then $h \circ \mu \in \operatorname{Hom}\left(K_{*}(B \Gamma), K_{*}(B)\right)$. By the UCT 8.2 , there is $x \in K_{0}(C(B \Gamma) \otimes B)$ such that

$$
h(\mu(z))=\langle x, z\rangle \quad \text { for all } \quad z \in K_{*}(B \Gamma) .
$$

Note that if $t \in \operatorname{Ext}\left(K_{*}(B \Gamma), K_{*+1}(B)\right)$, then $\langle x+t, z\rangle=\langle x, z\rangle$. Thus without any loss of generality we may assume that $x$ is almost flat. Therefore there exist a finite open cover $\mathcal{V}$ of $B \Gamma$, a decreasing sequence $\left(\varepsilon_{n}\right)$ of positive numbers converging to 0 and two sequences $\left(E_{n}^{ \pm}\right)$of bundles over $B \Gamma$ such that $E_{n}^{ \pm}$are $\left(\mathcal{V}, \varepsilon_{n}\right)$-flat and satisfies $x=\left[E_{n}^{+}\right]-\left[E_{n}^{-}\right]$for all $n$. By passing to barycentric subdivisions of the simplicial structure $\Lambda$ of $B \Gamma$ we may assume that the dual cover $\mathcal{C}_{\Lambda}$ refines the open cover $\mathcal{V}$. By Proposition 4.8 we may arrange that the coordinate bundles underlying the $\left(E_{n}^{ \pm}\right)$ are normalized.

Write $F_{n}^{ \pm}$for the fibers of $E_{n}^{ \pm}$; these are finitely generated projective Hilbert $B$-modules and therefore embed as direct summands of some $B^{k(n)}$. This gives full-corner embeddings $A_{n}^{ \pm}:=$ $L_{B}\left(F_{n}^{ \pm}\right) \subset M_{k(n)}(B)$. Using Proposition 4.6 and Proposition 5.6 we associate with $E_{n}^{ \pm}$quasirepresentations $\pi_{n}^{ \pm}: \Gamma \rightarrow \mathrm{U}\left(A_{n}^{ \pm}\right)$such that $\lim _{n \rightarrow \infty}\left\|\pi_{n}^{ \pm}(s t)-\pi_{n}^{ \pm}(s) \pi_{n}^{ \pm}(t)\right\|=0$ for all $s, t \in \Gamma$ and $\lim _{n \rightarrow \infty}\left\|\pi_{n}^{ \pm}\left(s^{-1}\right)-\pi_{n}^{ \pm}(s)^{*}\right\|=0$ for all $s \in \Gamma$. The sequences $\left(\pi_{n}^{ \pm}\right)$induce morphisms of groups $\Gamma \rightarrow U\left(A_{\infty}^{ \pm}\right)$and hence $*$-homomorphisms $\pi_{\infty}^{ \pm}: \ell^{1}(\Gamma) \rightarrow A_{\infty}^{ \pm}$and $\bar{\pi}_{\infty}^{ \pm}: C^{*}(\Gamma) \rightarrow A_{\infty}^{ \pm}$where $A_{\infty}^{ \pm}=\prod_{n=1}^{\infty} A_{n}^{ \pm} / \sum_{n=1}^{\infty} A_{n}^{ \pm}$. Let $\bar{\pi}^{ \pm}: C^{*}(\Gamma) \rightarrow \prod_{n=1}^{\infty} A_{n}^{ \pm}$be a set-theoretic lifting of $\pi_{\infty}^{ \pm}$. Write $\bar{\pi}^{ \pm}=\left(\bar{\pi}_{n}^{ \pm}\right)_{n}$. For a sufficiently multiplicative quasi-representation $\pi: \Gamma \rightarrow \mathrm{U}(A)_{\delta}$ and a sufficiently small $\delta>0$, we will denote by $E_{\pi}$ the corresponding almost flat bundle constructed using the cocycle $\beta(\pi)$ constructed in Proposition 5.4 (see Notation 8.3). For $n$ sufficiently large we have that $\left[E_{\pi_{n}^{ \pm}}\right]=\left[E_{n}^{ \pm}\right]$. This follows from Theorem 3.3 and Proposition 7.2 since bundles whose cocycles are sufficiently close to each other are isomorphic.

Let us recall that the full assembly map $\mu: K_{*}(B \Gamma) \rightarrow K_{*}\left(C^{*}(\Gamma)\right)$ is implemented by the Mishchenko line bundle $\ell \in K_{0}\left(C(B \Gamma) \otimes C^{*}(\Gamma)\right)$, via the Kasparov product

$$
K K\left(\mathbb{C}, C(B \Gamma) \otimes C^{*}(\Gamma)\right) \times K K_{*}(C(B \Gamma), \mathbb{C}) \rightarrow K K_{*}\left(\mathbb{C}, C^{*}(\Gamma)\right),
$$


$(\ell, z) \mapsto \mu(z):=\langle\ell, z\rangle$.

We have seen earlier (8.1) that one can represent $\ell$ by a projection $e$ in matrices over $C(B \Gamma) \otimes$ $\mathbb{C}[\Gamma]$. So long as $n$ is sufficiently large, Proposition 8.4 guarantees that $\left[E_{\pi_{n}^{ \pm}}\right]$equals $\ell_{\pi_{n}^{ \pm}}$, the pushforward of $e$ by $\operatorname{id}_{C(B \Gamma)} \otimes \pi_{n}^{ \pm}$in $K_{0}\left(C(B \Gamma) \otimes A_{n}^{ \pm}\right) \cong K_{0}(C(B \Gamma) \otimes B)$. The latter isomorphism is induced by the full-corner embeddings $A_{n}^{ \pm} \subset M_{k(n)}(B)$. It follows that

$$
\langle x, z\rangle=\left\langle\left[E_{n}^{+}\right], z\right\rangle-\left\langle\left[E_{n}^{-}\right], z\right\rangle=\left\langle\ell_{\pi_{n}^{+}}, z\right\rangle-\left\langle\ell_{\pi_{n}^{-}}, z\right\rangle
$$

for all $z \in K_{*}(B \Gamma)$. Let $\mu_{\ell^{1}}: K_{*}(B \Gamma) \rightarrow K_{*}\left(\ell^{1}(\Gamma)\right)$ be Lafforgue's $\ell^{1}$-version of the assembly map. It is known that $j_{*} \circ \mu_{\ell^{1}}=\mu$ where $j: \ell^{1}(\Gamma) \rightarrow C^{*}(\Gamma)$ is the canonical map 15. By [4. Theorem 3.2 and Corollary 3.5] we have that

$$
\left\langle\ell_{\pi_{n}^{ \pm}}, z\right\rangle=\pi_{n_{\sharp}}^{ \pm}\left(\mu_{\ell^{1}}(z)\right)
$$

for each $z \in K^{*}(B \Gamma)$ so long as $n$ is sufficiently large. (For $z \in K_{0}(B \Gamma)$ we interpret $\pi_{n \sharp}^{ \pm}\left(\mu_{\ell^{1}}(z)\right.$ ) as $\pi_{n_{\sharp}}^{ \pm}\left(p_{z}\right)-\pi_{n \sharp}^{ \pm}\left(q_{z}\right)$ where $p_{z}, q_{z}$ are projections with $\mu_{\ell^{1}}(z)=\left[p_{z}\right]-\left[q_{z}\right]$. There is a similar interpretation of $\pi_{n \sharp}^{ \pm}\left(\mu_{\ell^{1}}(z)\right)$ for $z \in K_{1}(B \Gamma)$ obtained by replacing idempotents by invertible elements.) Therefore,

$$
\left(\pi_{n \sharp}^{+}\left(\mu_{\ell^{1}}(z)\right)-\pi_{n \sharp}^{-}\left(\mu_{\ell^{1}}(z)\right)\right) \equiv(\langle x, z\rangle)=(h(\mu(z)))
$$

for all $z \in K_{*}(B \Gamma)$. From Remark $8.2(3)$ we deduce that

$$
\begin{gathered}
\bar{\pi}_{n \sharp}^{ \pm}(\mu(z))=\bar{\pi}_{n \sharp}^{ \pm}\left(j_{*}\left(\mu_{\ell^{1}}(z)\right)\right) \equiv \pi_{n \sharp}^{ \pm}\left(\mu_{\ell^{1}}(z)\right) \\
\left(\bar{\pi}_{n \sharp}^{+}(\mu(z))-\bar{\pi}_{n \sharp}^{-}(\mu(z))\right) \equiv(h(\mu(z)))
\end{gathered}
$$

for all $z \in K_{*}(B \Gamma)$. The discrete asymptotic homomorphisms $\left\{\bar{\pi}_{n}^{ \pm}: C^{*}(\Gamma) \rightarrow M_{k(n)}(B)\right\}_{n}$ have the desired properties.

$(2) \Rightarrow(1)$. Let us assume now that $K_{*}(B)$ is finitely generated and that $\mu$ is split injective. Let $x \in K_{0}(C(B \Gamma) \otimes B)$ be given. We will find an almost flat element $y \in K_{0}(C(B \Gamma) \otimes B)$ such that $x-y \in \operatorname{Ext}\left(K_{*}(B \Gamma), K_{*+1}(B)\right)$. Since $\mu$ is split-injective by hypothesis (i.e. the image of $\mu$ is a direct summand of $K_{*}\left(C^{*}(\Gamma)\right)$, it follows from the exactness of the sequence (8.2) that there is a homomorphism $h: K_{*}\left(C^{*}(\Gamma)\right) \rightarrow K_{*}(B)$ such that $h \circ \mu(z)=\langle x, z\rangle$ for all $z \in K_{*}(B \Gamma)$. By the assumptions in (2) there are two discrete asymptotic homomorphisms $\left\{\pi_{n}^{ \pm}: C^{*}(\Gamma) \rightarrow M_{k(n)}(B)\right\}_{n}$ such that

$$
\left(\pi_{n \sharp}^{+}(\mu(z))-\pi_{n \sharp}^{-}(\mu(z))\right) \equiv(h(\mu(z)))=(\langle x, z\rangle)
$$

for all $z \in K^{0}(B \Gamma)$. By a standard perturbation argument we may assume that $\pi_{n}^{ \pm}(s) \in U_{k(n)}(B)$ for all $n$ and $s \in \Gamma$. Invoking [4, Thm. 3.2 and Cor. 3.5] we obtain that

$$
\left(\left\langle\ell_{\pi_{n}^{ \pm}}, z\right\rangle\right) \equiv\left(\pi_{n \sharp}^{ \pm}(\mu(z))\right) .
$$

By Proposition 8.4 we have that $\ell_{\pi_{n}^{ \pm}}=\left[E_{\pi_{n}^{ \pm}}\right]$where the bundles $E_{\pi_{n}^{ \pm}}$are $\left(\mathcal{V}, \varepsilon_{n}\right)$-flat and $\varepsilon_{n} \rightarrow 0$. If we set $x_{n}:=\left[E_{\pi_{n}^{+}}\right]-\left[E_{\pi_{n}^{-}}\right]$, it follows from 8.3) that for any $z \in K_{*}(B \Gamma)$, there is $n_{z}$ such that $\langle x, z\rangle=\left\langle x_{n}, z\right\rangle$ for $n \geq n_{z}$. Since $K_{*}(B \Gamma)$ is finitely generated there exists $n_{0}$ such that $x-x_{n} \in H:=\operatorname{Ext}\left(K_{*}(B \Gamma), K_{*+1}(B)\right)$ for all $n \geq 0$. Since $K_{*}(B)$ is finitely generated, the group $H$ is finite. Therefore after passing to a subsequence of $\left(x_{n}\right)$ we may arrange that the sequence $\left(x-x_{n}\right)$ is constant and so there is $t \in H$ such that $x+t=x_{n}$ for all $n$. It follows that $y:=x+t$ is is almost flat and $x-y \in H$. 
If $B \Gamma$ is a finite complex and the group $K_{*}(B)$ is finitely generated and torsion-free, it follows that the group $\operatorname{Ext}\left(K_{*}(B \Gamma), K_{*+1}(B)\right)$ is finite and by $(8.2)$ it coincides with the torsion subgroup of $K_{0}(C(B \Gamma) \otimes B)$. By taking $B=\mathbb{C}$ in Theorem 8.6 we obtain the following.

8.7 Theorem. Let $\Gamma$ be a discrete countable group whose classifying space $B \Gamma$ is a finite simplicial complex. If the full assembly map is bijective, then the following conditions are equivalent

(1) All elements of $K^{0}(B \Gamma)$ are almost flat modulo torsion.

(2) For any group homomorphism $h: K_{0}\left(C^{*}(\Gamma)\right) \rightarrow \mathbb{Z}$ there exist discrete asymptotic homomorphisms $\left\{\pi_{n}^{ \pm}: C^{*}(\Gamma) \rightarrow M_{k(n)}(\mathbb{C})\right\}_{n}$ such that $\left(\pi_{n \sharp}^{+}(y)-\pi_{n \sharp}^{-}(y)\right) \equiv(h(y))$ for every $y \in$ $K_{0}\left(C^{*}(\Gamma)\right)$.

With the terminology from the introduction, condition (2) above amounts to saying that $C^{*}(\Gamma)$ is K-theoretically MF.

8.8 Remark. Gromov indicates in 7,8 how one constructs nontrivial almost flat $K$-theory classes for residually finite groups $\Gamma$ that are fundamental groups of even dimensional non-positively curved compact manifolds.

\section{References}

[1] Bruce Blackadar and Eberhard Kirchberg, Generalized inductive limits of finite-dimensional $C^{*}$-algebras, Math. Ann. 307 (1997), no. 3, 343-380. MR 1437044 (98c:46112)

[2] José R. Carrión and Marius Dadarlat, Quasi-representations of surface groups, J. Lond. Math. Soc. (2) 88 (2013), no. 2, 501-522. MR 3106733

[3] Alain Connes, Mikhail Gromov, and Henri Moscovici, Conjecture de Novikov et fibrés presque plats, C. R. Acad. Sci. Paris Sér. I Math. 310 (1990), no. 5, 273-277. MR 1042862

[4] Marius Dadarlat, Group quasi-representations and index theory, J. Topol. Anal. 4 (2012), no. 3, 297-319. MR 2982445

[5] _ Group quasi-representations and almost flat bundles, J. Noncommut. Geom. 8 (2014), no. 1, 163-178. MR 3275029

[6] James Dugundji, An extension of Tietze's theorem, Pacific J. Math. 1 (1951), 353-367. MR 0044116

[7] Mikhael Gromov, Geometric reflections on the Novikov conjecture, Novikov conjectures, index theorems and rigidity, Vol. 1 (Oberwolfach, 1993), London Math. Soc. Lecture Note Ser., vol. 226, Cambridge Univ. Press, Cambridge, 1995, pp. 164-173. MR 1388301

[8] _ Positive curvature, macroscopic dimension, spectral gaps and higher signatures, Functional analysis on the eve of the 21st century, Vol. II (New Brunswick, NJ, 1993), Progr. Math., vol. 132, Birkhäuser Boston, Boston, MA, 1996, pp. 1-213. MR 1389019

[9] Mikhael Gromov and H. Blaine Lawson, Jr., Positive scalar curvature and the Dirac operator on complete Riemannian manifolds, Inst. Hautes Études Sci. Publ. Math. (1983), no. 58, 83196 (1984). MR 720933 
[10] Bernhard Hanke and Thomas Schick, Enlargeability and index theory, J. Differential Geom. 74 (2006), no. 2, 293-320. MR 2259056

[11] Nigel Higson and Gennadi G. Kasparov, E-theory and KK-theory for groups which act properly and isometrically on Hilbert space, Invent. Math. 144 (2001), no. 1, 23-74. MR 1821144

[12] Max Karoubi, K-theory, Springer-Verlag, Berlin, 1978, An introduction, Grundlehren der Mathematischen Wissenschaften, Band 226. MR 0488029

[13] Gennadi G. Kasparov, Equivariant KK-theory and the Novikov conjecture, Invent. Math. 91 (1988), no. 1, 147-201. MR 918241

[14] Gennadi G. Kasparov and Georges Skandalis, Groups acting on buildings, operator K-theory, and Novikov's conjecture, K-Theory 4 (1991), no. 4, 303-337. MR 1115824

[15] Vincent Lafforgue, $K$-théorie bivariante pour les algèbres de Banach et conjecture de BaumConnes, Invent. Math. 149 (2002), no. 1, 1-95. MR 1914617

[16] Vladimir M. Manuilov and Aleksandr S. Mishchenko, Almost, asymptotic and Fredholm representations of discrete groups, Acta Appl. Math. 68 (2001), no. 1-3, 159-210, Noncommutative geometry and operator $K$-theory. MR 1865957

[17] Alexander S. Mishchenko and Nicolae Teleman, Almost flat bundles and almost flat structures, Topol. Methods Nonlinear Anal. 26 (2005), no. 1, 75-87. MR 2179351

[18] Henri Moscovici, Cyclic cohomology and invariants of multiply connected manifolds, Proceedings of the International Congress of Mathematicians, Vol. I, II (Kyoto, 1990) (Tokyo), Math. Soc. Japan, 1991, pp. 675-688. MR 1159254

[19] Anthony Phillips and David Stone, Lattice gauge fields, principal bundles and the calculation of topological charge, Comm. Math. Phys. 103 (1986), no. 4, 599-636. MR 832541

[20] Anthony V. Phillips and David A. Stone, The computation of characteristic classes of lattice gauge fields, Comm. Math. Phys. 131 (1990), no. 2, 255-282. MR 1065672

[21] Georges Skandalis, Approche de la conjecture de Novikov par la cohomologie cyclique (d'après A. Connes, M. Gromov et H. Moscovici), Astérisque (1991), no. 201-203, Exp. No. 739, 299320 (1992), Séminaire Bourbaki, Vol. 1990/91. MR 1157846

[22] Edwin H. Spanier, Algebraic topology, McGraw-Hill Book Co., New York, 1966. MR 0210112

[23] Jean-Louis Tu, The gamma element for groups which admit a uniform embedding into Hilbert space, Recent advances in operator theory, operator algebras, and their applications, Oper. Theory Adv. Appl., vol. 153, Birkhäuser, Basel, 2005, pp. 271-286. MR 2105483

[24] Guoliang Yu, The coarse Baum-Connes conjecture for spaces which admit a uniform embedding into Hilbert space, Invent. Math. 139 (2000), no. 1, 201-240. MR 1728880 